\title{
Trends in food and nutritional intakes of French adults from 1999 to 2007: results from the INCA surveys
}

\author{
Carine Dubuisson ${ }^{1}$, Sandrine Lioret ${ }^{1}$, Mathilde Touvier $^{1}$, Ariane Dufour ${ }^{1}$, Gloria Calamassi-Tran ${ }^{1}$, \\ Jean-Luc Volatier ${ }^{2}$ and Lionel Lafay ${ }^{1}$ \\ ${ }^{1}$ French Food Safety Agency (AFSSA), Direction of Risk Assessment for Nutrition and Food Safety, Office of Scientific Support for \\ Risk Assessment, Dietary Survey Unit - Nutritional Epidemiology, 27-31 Avenue du Général Leclerc, 94701 Maisons-Alfort \\ Cedex, France \\ ${ }^{2}$ French Food Safety Agency (AFSSA), Office of Scientific Support for Risk Assessment, Maisons-Alfort, France
}

(Received 16 February 2009 - Revised 16 September 2009 - Accepted 29 September 2009 - First published online 23 December 2009)

Two independent cross-sectional dietary surveys (the Individual and National Food Consumption Surveys, INCA), performed in 1998-99 (INCA1) and in 2006-07 (INCA2) on nationally representative samples of French people, were used to analyse trends in the dietary habits and nutritional intake of French adults. Food consumption was recorded through 7-d dietary records, and nutritional intakes were assessed using the French food composition database. After exclusion of under-reporters, analyses were performed on 3267 adults, aged 18-79 years: 1345 from INCA1 and 1922 from INCA2. The trends highlighted over the 8-year period showed a decrease in consumption of dairy products, meat, bread, potatoes, pastries/croissant-like pastries/cakes/biscuits and sugar/confectionery. In contrast, the consumption of fruits and vegetables, rice, ice cream and chocolate increased. Other food groups, like fish and snacking foods, remained stable. Food choices were mostly age specific. These age differences remained consistent over the years and underlined two opposite dietary trends: a 'traditional' one mainly followed by the elderly, and a 'snacking and convenience' one mainly adopted by young adults. The overall trends in food consumption did not influence the mean energy intake, but did slightly modify the contribution of each macronutrient to energy intake. These repeated surveys highlighted the fact that trends in French food habits have moved towards an average European diet at the crossroads between Mediterranean and Northern diets, and that food consumption changes impacted, to a lesser extent, nutritional intake.

Trends: INCA surveys: Food consumption: Nutritional intake: France

With the growing global concern about chronic diet-related diseases such as obesity and CVD, the WHO and the FAO have stressed the importance of a balanced diet to prevent these diseases ${ }^{(1)}$. In France, a national nutrition policy was established in January 2001, through the First National Nutrition and Health Program, followed by Second National Nutrition and Health Program $(2006-10)^{(2)}$. As in many countries $^{(3-5)}$, this policy was supported by food-based dietary guidelines, available for the general population and age subgroups (adults, children, elderly, etc.), and mass media campaigns to promote healthy dietary habits and physical activity. In other respects, the food habits in Western countries have tended to converge towards a 'Western diet': in Europe, traditional national diets have become less distinct within the last 40 years $^{(6)}$. Indeed, the Mediterranean countries have increased their saturated fat, cholesterol and sugar intakes, whereas some Northern European countries have lowered their sugar and fat intakes ${ }^{(6,7)}$. France is situated at the crossroads of these European diets and shows strong regional dietary behaviours that remain consistent for the generations ${ }^{(8)}$.
These fast changes in dietary habits and the nutrition policies implemented to establish healthier food behaviours highlight the need for monitoring food habits and nutritional intake over time. To ensure the validity of trends observed in the period between two different studies, both should use similar methodologies (population scale, food assessment method, season, etc.); otherwise, the findings could reflect only methodological differences ${ }^{(9,10)}$. Some countries have already monitored dietary habits by repeating cross-sectional studies $^{(11-16)}$. However, to our knowledge, no comparable data are available at a national level in France.

In 2006-07, the French Food Safety Agency (Agence française de sécurité sanitaire des aliments, AFSSA) carried out a nationally representative food consumption survey (Individual and National Food Consumption Surveys, INCA2), with a population scale and dietary assessment methodology similar to the previous INCA1 survey conducted in 1998-99. This similarity allows monitoring of eating behaviours and food intake over time. Thus, the main objective of the present study was to investigate the trends in food consumption and

Abbreviations: AFSSA, Agence française de sécurité sanitaire des aliments; INCA, Individual and National Food Consumption Surveys; INSEE, French National Institute of Statistics and of Economic Studies.

* Corresponding author: Carine Dubuisson, fax +3314977 38 92, email c.dubuisson@afssa.fr 
nutritional intake of French adults in the period between the INCA surveys.

\section{Materials and methods}

Data from the two French national cross-sectional food consumption surveys, INCA1 (1998-99) and INCA2 (2006-07), were used in the analysis. Both surveys were performed on nationally representative samples of French people and designed to assess food intake patterns.

\section{Subjects}

The French INCA1 survey methodology has been described elsewhere ${ }^{(17,18)}$. Briefly, this survey was performed between August 1998 and June 1999 by the Research Centre for the Study and the Observation of Living Conditions and the French Food Safety Agency (AFSSA). Two independent samples, composed of adults aged 15 years and over and children aged 3-14 years, were made representative of the French population through stratification (region of residence and size of urban area) and use of the quota method (age, sex, household size and socio-professional status of head of household ${ }^{(19)}$. Food records and questionnaires were completed by 1985 adults, of whom 1849 were aged 18-79 years.

The French INCA2 food consumption survey was carried out between December 2005 and May 2007 by AFSSA. Two independent random samples of 3- to 17-year-old children and 18- to 79-year-old adults were drawn using a multistage cluster sampling technique. The sampling frame was extracted from the national census published by French National Institute of Statistics and of Economic Studies (INSEE). First, 181 primary geographical units, stratified by region of residence and size of urban area, were randomly selected with probability proportional to size. Then, households were randomly drawn within each primary sampling unit, and two independent sampling frames were set up: the first restricted to households including at least one child and the second included households with or without children. Finally, within each household, either a child or an adult was randomly selected.

Information letters were sent to the households and were followed by a phone call when the telephone number was available (23\% for the adults' database). During this phone call, more information about the study and its objectives was provided, and the eligible person was selected. If the subject agreed to participate in the survey, an appointment at home was made. When the telephone number was not available or when the household head was 65 years old or over, trained investigators had gone directly to the households. Data on 2624 adults aged 18-79 years were thus collected, which correspond to a response rate of $63 \%$ for the adult sample.

Under-reporters were identified in both studies by comparing the reported energy intake to the $\mathrm{BMR}$ as estimated from the Schofield equations ${ }^{(20)}$, and a cut-off value for physical activity level was fixed at $1 \cdot 55^{(21)}$. This excluded 504 adults $(27.3 \%)$ and 702 adults $(26.8 \%)$ from the INCA1 and INCA2 survey datasets, respectively. These rates of under-reporting were similar to those usually observed in dietary surveys ${ }^{(22-24)}$. Analyses were thus performed on 3267 subjects: 1345 from INCA1 and 1922 from INCA2.

\section{Measurements}

In both the INCA1 and INCA2 surveys, diet was assessed using a 7-d open-ended food record. Each day of the food record was divided into three main meals (breakfast, lunch and dinner) and three between-meals snacks. The subjects were asked to describe as precisely as possible all food and beverage intakes for seven consecutive days: food name, origin (home-made or industrial product) and features (low fat, low sugar, fortified, dietetic, as well as fresh, canned or frozen). Portion sizes were estimated using the Supplémentation en Vitamines et en Minéraux Antioxydants photographic booklet ${ }^{(25)}$ or expressed by weight or household measures (spoon). In INCA2 only, table and cooking uses of salt, fat and sugar were also recorded in the self-reported questionnaire. Added fat and sugar were then integrated into the INCA2 food record, whereas salt was not. Average daily nutritional intakes were evaluated using the French food composition databases $^{(26,27)}$ from each survey period (1998 and 2006, respectively). The individual behavioural, demographical and socio-economic variables were collected using self-reported and face-to-face questionnaires.

During the first face-to-face interview, the 7-d record and a self-administered questionnaire were delivered at home by a trained and certified investigator, who explained to the subjects how to complete them. Just after the survey week, the investigator came back and checked the accuracy of the information reported in both documents. The subject was included when at least $3 \mathrm{~d}$ of the diary were filled in, but most of the participants completed all $7 \mathrm{~d}$ (96\% in INCA2). An additional face-to-face questionnaire, including questions on socioeconomic status, was administered. For the INCA2 survey, interviewers measured participant weight and height, but these figures were self-reported in the INCA1 survey.

The studies were approved by the French Data Protection Authority (Commission Nationale Informatique et Libertés).

\section{Data analysis}

All analyses were computed on Statistical Analysis System software version 9.1 (SAS Institute Inc., Cary, NC, USA). Age, sex and household head occupational category in the INCA1 sample compared satisfactorily with the national census for 1997 published by INSEE, despite a slight overrepresentation of women and young adults. INCA2 survey data were weighted for unequal sampling probabilities and for differential non-responses by region, agglomeration size, age, sex, occupation of the household head, size of the household and season. The external data used came from the INSEE national dataset for 2005 .

Age was divided into a three-class variable (18-34, 35-54 and 55-79 years) and individual occupational status was classified in 'high' (executive, top-management and professional categories), 'medium' (employees, technicians and similar), 'low' (manual workers) and 'inactive' (retired, students, unemployed and housewives/house husbands).

The average food intake (g/d) of thirty-eight food groups was estimated in both surveys. The results were stratified by sex and age to take into account the slight over-representation of women and young adults in INCA1. To enable comparisons of the food group consumption, the INCA1 data were recoded according to 
the food group nomenclature used for the INCA2 survey. The consumer rate for each food group was also assessed. It represented the proportion of people who consumed at least one food (or beverage) belonging to the food group during the $7 \mathrm{~d}$ of the survey. The average dietary intakes in energy $(\mathrm{MJ} / \mathrm{d})$, carbohydrates ( $\mathrm{g} / \mathrm{d}$ and \% energy), proteins ( $\mathrm{g} / \mathrm{d}$ and $\%$ energy), lipids (g/d and \% energy), SFA (g/d), MUFA (g/d), PUFA $(\mathrm{g} / \mathrm{d})$, sugar $(\mathrm{g} / \mathrm{d})$, polysaccharides $(\mathrm{g} / \mathrm{d})$, fibre $(\mathrm{g} / \mathrm{d})$, alcohol (g/d), Na (mg/d), Ca (mg/d), Fe (mg/d), vitamin C $(\mathrm{mg} / \mathrm{d})$ and folate $(\mu \mathrm{g} / \mathrm{d})$ were also estimated in both surveys. Trends between the two surveys were calculated by:

$$
\frac{(\text { INCA2 value }- \text { INCA1 value })}{\text { INCA1 value }} \times 100(\text { in } \%) .
$$

The differences in means were tested with ANOVA and consumer rates were compared with $\chi^{2}$ tests. A $P$-value of 0.05 was used as the threshold for significance. Consumption of fats and beverages, as well as fatty acids, could not be compared between the two surveys because of methodological improvements in their recording in INCA2. On the contrary, since salt collected in the self-reported questionnaire in INCA2 was not taken into account, the salt intake derived from foods could be compared between the two studies.

\section{Results \\ Description of the subjects}

The general characteristics of the subjects are presented for each INCA survey in Table 1. The mean age of the samples increased between the two surveys from 44.6 (SE 0.4) to 46.4 (SE 0.4) years. The level of occupational status also increased, with more subjects in the high level of occupational status and fewer inactives. INCA2 included less households composed of five or more people than INCA1. Finally, the geographic distribution of the subjects was similar between the two surveys.

Trends in food consumption between 1998-99 and 2006-07

The thirty-eight food groups in the analysis are described in Table 2. The consumer rates and absolute amounts of food intake for these food groups are presented by sex-age subgroups for the two surveys in Table 3. Over the 8-year span, several trends in food consumption by French adults can be highlighted.

Trends in starchy food consumption. Starchy foods included bread, potatoes, pasta, rice, wheat and pulses. Traditional starchy foods, like bread and potatoes, were partly replaced by pasta, rice and wheat. No significant trends were noted for pulses consumption. The strongest changes concerned young men and women with bread $(-20.7$ and $-13.2 \%$, respectively) and young men for potatoes $(-15.8 \%)$, as well as rice and wheat $(+43.8 \%)$. However, the overall consumption of starchy foods remained constant between the INCA1 and INCA2 survey datasets in all sex-age subgroups. Moreover, the age group preferences in starchy foods consumption observed in 1999 were reinforced in 2007: the elderly ate more bread and young people ate more pasta, rice and wheat.

Trends in sweetened food consumption. Sweetened foods consisted of croissant-like pastries/other sweetened pastries/ cakes/biscuits, ice cream/frozen desserts, chocolate and sugar/confectionery. Between the two surveys, consumption

Table 1. Characteristics of adults sample in the Individual and National Food Consumption Surveys (INCA1 and INCA2) (under-reporters excluded)

(Mean values with their standard errors)

\begin{tabular}{|c|c|c|c|c|c|}
\hline & \multicolumn{2}{|c|}{ INCA1 } & \multicolumn{2}{|c|}{ INCA2* $^{*}$} & \multirow[b]{2}{*}{$P$} \\
\hline & Mean (\%) & $\mathrm{SE}$ & Mean (\%) & SE & \\
\hline$n$ & 1345 & & 1922 & & \\
\hline Men & $45 \cdot 6$ & 1.4 & $51 \cdot 1$ & 1.3 & 0.005 \\
\hline \multicolumn{6}{|l|}{ Age group } \\
\hline $18-34$ years & $33 \cdot 2$ & 1.3 & $28 \cdot 7$ & 1.3 & \multirow[t]{3}{*}{0.003} \\
\hline $35-54$ years & $38 \cdot 3$ & 1.3 & $36 \cdot 7$ & 1.2 & \\
\hline $55-79$ years & $28 \cdot 6$ & $1 \cdot 2$ & $34 \cdot 6$ & $1 \cdot 2$ & \\
\hline \multicolumn{6}{|l|}{ Occupational status } \\
\hline High & $6 \cdot 4$ & 0.7 & $9 \cdot 6$ & 0.6 & \multirow[t]{4}{*}{0.0007} \\
\hline Medium & 33.0 & 1.3 & $35 \cdot 7$ & $1 \cdot 1$ & \\
\hline Low & 11.9 & 0.9 & $12 \cdot 7$ & 1.0 & \\
\hline Inactive (including unemployed) & $48 \cdot 7$ & 1.4 & 41.9 & $1 \cdot 3$ & \\
\hline \multicolumn{6}{|l|}{ Household size } \\
\hline One person & $13 \cdot 3$ & 0.9 & $13 \cdot 2$ & 0.7 & \multirow[t]{5}{*}{0.03} \\
\hline Two persons & $33 \cdot 2$ & $1 \cdot 3$ & $35 \cdot 4$ & $1 \cdot 1$ & \\
\hline Three persons & $18 \cdot 6$ & $1 \cdot 1$ & $20 \cdot 6$ & $1 \cdot 1$ & \\
\hline Four persons & $20 \cdot 1$ & $1 \cdot 1$ & $20 \cdot 1$ & $1 \cdot 1$ & \\
\hline Five or more persons & $14 \cdot 8$ & 1.0 & $10 \cdot 7$ & 0.7 & \\
\hline \multicolumn{6}{|l|}{ Region } \\
\hline Paris region & $20 \cdot 2$ & $1 \cdot 1$ & $17 \cdot 4$ & $1 \cdot 1$ & \multirow[t]{5}{*}{0.13} \\
\hline North-East & $23 \cdot 1$ & $1 \cdot 1$ & $21 \cdot 8$ & $1 \cdot 1$ & \\
\hline North-West & $22 \cdot 5$ & $1 \cdot 1$ & $25 \cdot 8$ & $1 \cdot 2$ & \\
\hline South-East & $22 \cdot 9$ & $1 \cdot 1$ & $22 \cdot 8$ & $1 \cdot 1$ & \\
\hline South-West & $11 \cdot 3$ & 0.9 & $12 \cdot 3$ & 0.9 & \\
\hline
\end{tabular}

${ }^{*}$ Weighted results. 
Table 2. Description of the thirty-eight food groups

\begin{tabular}{|c|c|}
\hline Food groups & Description \\
\hline Bread and bread products & Breads (white, wholemeals, etc.), rusk and other bread products \\
\hline Pasta & / \\
\hline Rice and wheat & Brown and white rice, semolina and wheat \\
\hline Potatoes & $\begin{array}{l}\text { Potatoes, sweet potatoes, potato crisps, dauphine potatoes, potato chips, } \\
\text { mashed potatoes prepared from flakes or fresh }\end{array}$ \\
\hline Pulses & Broad beans, haricot beans, kidney beans, lentils, split peas, chick peas and tofu \\
\hline $\begin{array}{l}\text { Croissant-like pastries, other sweetened pastries, } \\
\text { cakes and biscuits }\end{array}$ & $\begin{array}{l}\text { Brioche, croissants, croissants with chocolate filling, milk bread rolls, cakes, } \\
\text { pastries, pies, fritters, crepes, waffles and preparations for pastries, shortbread } \\
\text { biscuits, chocolate biscuits, flaky pastry, biscuits with fruit or jam filling, butter biscuits, } \\
\text { wafers, meringue, cookies etc. }\end{array}$ \\
\hline Ice cream and frozen desserts & Ice cream, frozen desserts and sherbets \\
\hline Chocolate & Chocolate, chocolate candy bars and chocolate hazelnut spread \\
\hline Sugar and confectionery & Sugars, marmalades, jams, honey and non-chocolate confectionery \\
\hline Milk & Whole, semi-skimmed and skimmed milks, condensed milks and evaporated milks \\
\hline Cheeses & $\begin{array}{l}\text { Firm, hard, stretched or brined cheeses, soft cheeses with moulded rind, soft cheeses } \\
\text { with washed rind, blue cheeses, processed cheeses, uncured cheeses, etc. }\end{array}$ \\
\hline Other dairy products & Cream, yoghurt, cottage cheese and petit suisses \\
\hline Cream desserts & Custard desserts and jellified milks \\
\hline Butter & 1 \\
\hline Oil & / \\
\hline Margarines & I \\
\hline Other fats & Peanut butter, goose or duck fat \\
\hline Eggs and egg products & Fried, scrambled, raw, hard-boiled and poached eggs and omelettes \\
\hline Meat & Lamb, beef, veal and pork meats \\
\hline Poultry and games & Chicken, turkey, duck meats and game \\
\hline Offal & Brain, heart, liver, tongue, sweetbreads and kidneys \\
\hline Meat products & Raw and cooked hams, sausages, pâtés and rillettes \\
\hline Fish & Raw, canned and cooked fish and fish-based products \\
\hline Crustaceans and molluscs & / \\
\hline Vegetables & Raw and cooked vegetables \\
\hline Fruit & Raw fruit \\
\hline Mashed and cooked fruit & i \\
\hline Dried fruit, nuts and seeds & I \\
\hline Breakfast cereals & l \\
\hline Pizza, savoury pastries & Pizzas, quiches, savoury pies and puff pastries \\
\hline Sandwiches and hamburgers & $\begin{array}{l}\text { Grilled cheese, ham and egg sandwiches, grilled ham and cheese sandwiches, hamburgers, } \\
\text { hot dogs, sandwiches on French bread, pita bread, loaf bread and panini bread }\end{array}$ \\
\hline Soups & Ready-to-eat and home-made soups, instant soups and stocks \\
\hline Mixed dishes & $\begin{array}{l}\text { Meat-based dishes, savoury fritters and crepes, pasta- and potato-based dishes, } \\
\text { cheese-based dishes, vegetable-based dishes and side dishes }\end{array}$ \\
\hline Waters & Plain and carbonated mineral waters, tap water and spring water \\
\hline Non-alcoholic beverages & Fruit juices, nectars, fruit-based beverages and soft drinks \\
\hline Alcoholic beverages & Wine, beer, hard cider, liquor, cocktails and alcoholic blended spirits \\
\hline Coffee & / \\
\hline Other hot beverages & Cocoa or chocolate beverages, chicory coffee, tea and herbal tea \\
\hline
\end{tabular}

of croissant-like pastries/other sweetened pastries/cakes/biscuits and sugar/confectionery decreased. Conversely, ice cream and chocolate consumption increased but still remained low. The higher consumption of chocolate was mainly due to a higher consumer rate. For ice cream, this rate strongly increased in older women $(+70.8 \%)$ and decreased in young men $(-34.0 \%)$. Overall, the consumption of sweetened foods tended to decrease, particularly in older men $(-16.7 \%)$ and young women $(-9.8 \%)$, but remained higher in the youngest, except for sugar/confectionery, which were still more consumed by the elderly.

Trends in dairy product consumption. Between 1999 and 2007, milk and cheese consumption strongly dropped, particularly for women $(-21.5$ to $-37.4 \%$ for milk and -19.1 to $-24.9 \%$ for cheese, according to age category) and older men $(-27.9$ and $-23.8 \%$, respectively). It occurred along with an overall fall, up to $-30 \%$, of the consumer rate for milk. Conversely, the intake of other dairy products (yoghurt and cottage cheese) increased, except in the youngest adults. Overall, intake of dairy products (milk, cheese and other dairy products) in old and young women fell significantly ( -20.3 and $-23.6 \%$, respectively), but remained stable in men. In 2007, as in 1999, milk was consumed in higher amounts by the youngest compared with the oldest adults, and the reverse was observed for cheese. No other age differences existed for other dairy product consumption in INCA2.

Trends in meat, fish and egg product consumption. Meat (red meat and poultry) intake strongly decreased in women, as did egg intake in men, along with a lower consumption rate. In parallel, meat product intake of young women decreased $(-18 \cdot 3 \%)$. Only fish intake remained unchanged. Overall, the consumption of meat (all kinds), fish and sea products and egg products decreased between the two studies, particularly in women $(-6.5$ to $-14.4 \%$ by age category) and in middle-aged men $(-9.4 \%)$. Most of the age-specific dietary habits observed in the INCA1 survey remained in 
Table 3. Trends in percentage of food group consumers (\%) and food group consumption ( $\mathrm{g} / \mathrm{d}$ ), by sex and age, between Individual and National Food Consumption Surveys (INCA1, 1998-99 and INCA2, 2006-07) (under-reporters excluded)

(Mean values and standard deviations)

\begin{tabular}{|c|c|c|c|c|c|c|c|c|c|c|c|c|}
\hline \multirow[b]{2}{*}{ Food group } & \multirow[b]{2}{*}{ Sex } & \multirow[b]{2}{*}{ Age } & \multicolumn{4}{|c|}{ INCA1 (1998-99) } & \multicolumn{4}{|c|}{ INCA2 (2006-07) } & \multicolumn{2}{|c|}{ Trends } \\
\hline & & & $n$ & $\%$ & Mean & SD & $n$ & $\%$ & Mean & SD & $\%$ & Mean (\%) \\
\hline \multirow{6}{*}{ Bread and bread products } & \multirow{3}{*}{ Men } & $18-34$ years & 180 & $96 \cdot 1$ & $127 \cdot 1$ & $91 \cdot 8$ & 178 & $96 \cdot 7$ & $100 \cdot 8$ & $92 \cdot 1$ & +0.7 & $-20 \cdot 7^{\star \star}$ \\
\hline & & $35-54$ years & 245 & 98.4 & $160 \cdot 8$ & $115 \cdot 5$ & 354 & $99 \cdot 2$ & 146.9 & $81 \cdot 0$ & +0.9 & -8.7 \\
\hline & & $55-79$ years & 188 & 98.9 & $184 \cdot 4$ & $107 \cdot 8$ & 308 & $98 \cdot 6$ & $162 \cdot 1$ & $112 \cdot 0$ & -0.3 & $-12 \cdot 1^{*}$ \\
\hline & \multirow[t]{3}{*}{ Women } & $18-34$ years & 266 & $95 \cdot 9$ & 84.9 & 68.0 & 280 & $98 \cdot 1$ & 73.7 & $56 \cdot 2$ & $+2 \cdot 4$ & $-13 \cdot 2^{*}$ \\
\hline & & $35-54$ years & 270 & $97 \cdot 8$ & $100 \cdot 3$ & $66 \cdot 4$ & 477 & $99 \cdot 6$ & 94.3 & $51 \cdot 1$ & $+1.9^{\star \star}$ & $-6 \cdot 0$ \\
\hline & & $55-79$ years & 196 & $96 \cdot 4$ & $114 \cdot 1$ & $86 \cdot 3$ & 325 & $99 \cdot 2$ & $106 \cdot 4$ & 55.4 & $+2 \cdot 8^{\star}$ & $-6 \cdot 8$ \\
\hline \multirow[t]{6}{*}{ Pasta } & \multirow[t]{3}{*}{ Men } & $18-34$ years & 180 & 78.9 & 49.4 & $46 \cdot 3$ & 178 & 83.0 & 61.9 & 71.6 & $+5 \cdot 2$ & $+25 \cdot 2$ \\
\hline & & $35-54$ years & 245 & $77 \cdot 6$ & 41.3 & 39.9 & 354 & $78 \cdot 1$ & 46.5 & 47.5 & +0.8 & $+12 \cdot 8$ \\
\hline & & $55-79$ years & 188 & $71 \cdot 3$ & 33.5 & $35 \cdot 2$ & 308 & $75 \cdot 5$ & $35 \cdot 2$ & 39.9 & $+6 \cdot 0$ & +4.9 \\
\hline & \multirow[t]{3}{*}{ Women } & $18-34$ years & 266 & $81 \cdot 2$ & 34.5 & 29.9 & 280 & 84.9 & $41 \cdot 0$ & 37.5 & +4.5 & +18.9 \\
\hline & & $35-54$ years & 270 & $78 \cdot 1$ & $32 \cdot 0$ & $33 \cdot 2$ & 477 & $76 \cdot 6$ & 28.9 & $24 \cdot 8$ & -1.9 & -9.6 \\
\hline & & $55-79$ years & 196 & 71.9 & $23 \cdot 7$ & $22 \cdot 4$ & 325 & $72 \cdot 9$ & 23.4 & $21 \cdot 3$ & $+1 \cdot 3$ & $-1 \cdot 0$ \\
\hline \multirow{6}{*}{ Rice and wheat } & \multirow{3}{*}{ Men } & $18-34$ years & 180 & $64 \cdot 4$ & $25 \cdot 0$ & $27 \cdot 9$ & 178 & $70 \cdot 7$ & 35.9 & $52 \cdot 3$ & +9.8 & $+43 \cdot 8^{\star}$ \\
\hline & & $35-54$ years & 245 & $62 \cdot 9$ & $24 \cdot 2$ & $29 \cdot 8$ & 354 & 71.7 & 33.3 & 43.0 & $+14 \cdot 1^{*}$ & $+37 \cdot 9^{\star \star}$ \\
\hline & & $55-79$ years & 188 & $45 \cdot 2$ & $16 \cdot 8$ & $26 \cdot 1$ & 308 & $54 \cdot 4$ & 22.5 & 39.9 & $+20 \cdot 2$ & +33.7 \\
\hline & \multirow{3}{*}{ Women } & $18-34$ years & 266 & 69.5 & $24 \cdot 7$ & $27 \cdot 0$ & 280 & $68 \cdot 9$ & $23 \cdot 2$ & $29 \cdot 1$ & -0.9 & $-6 \cdot 2$ \\
\hline & & $35-54$ years & 270 & $63 \cdot 3$ & $19 \cdot 4$ & $23 \cdot 2$ & 477 & $67 \cdot 4$ & $23 \cdot 3$ & $27 \cdot 9$ & +6.4 & +19.9 \\
\hline & & $55-79$ years & 196 & $41 \cdot 8$ & $12 \cdot 7$ & $20 \cdot 9$ & 325 & 58.5 & $16 \cdot 9$ & $23 \cdot 0$ & $+39 \cdot 9^{\star \star \star}$ & $+33 \cdot 6$ \\
\hline \multirow{6}{*}{ Potatoes } & \multirow{3}{*}{ Men } & $18-34$ years & 180 & $96 \cdot 1$ & 74.8 & $51 \cdot 0$ & 178 & $91 \cdot 0$ & 63.0 & $57 \cdot 7$ & -5.4 & $-15 \cdot 8^{\star}$ \\
\hline & & $35-54$ years & 245 & $94 \cdot 7$ & $67 \cdot 7$ & $46 \cdot 8$ & 354 & 93.5 & $62 \cdot 8$ & $47 \cdot 8$ & -1.3 & $-7 \cdot 2$ \\
\hline & & $55-79$ years & 188 & $94 \cdot 7$ & $73 \cdot 2$ & $54 \cdot 2$ & 308 & $92 \cdot 3$ & 69.4 & $65 \cdot 7$ & -2.5 & $-5 \cdot 2$ \\
\hline & \multirow{3}{*}{ Women } & $18-34$ years & 266 & $93 \cdot 2$ & $56 \cdot 2$ & $41 \cdot 3$ & 280 & 93.1 & $52 \cdot 6$ & 43.0 & -0.1 & -6.5 \\
\hline & & $35-54$ years & 270 & 91.5 & $49 \cdot 8$ & $37 \cdot 7$ & 477 & 88.0 & $49 \cdot 0$ & $37 \cdot 1$ & -3.8 & -1.5 \\
\hline & & $55-79$ years & 196 & $88 \cdot 3$ & $61 \cdot 2$ & $50 \cdot 0$ & 325 & 88.2 & $51 \cdot 0$ & $42 \cdot 1$ & -0.1 & $-16 \cdot 6^{\star}$ \\
\hline \multirow{6}{*}{ Pulses } & Men & $18-34$ years & 180 & 29.4 & $9 \cdot 35$ & $16 \cdot 65$ & 178 & $27 \cdot 4$ & $9 \cdot 63$ & $25 \cdot 18$ & $-7 \cdot 1$ & $+3 \cdot 0$ \\
\hline & & $35-54$ years & 245 & $34 \cdot 3$ & $13 \cdot 17$ & $23 \cdot 77$ & 354 & 29.5 & 11.58 & $24 \cdot 71$ & -14.0 & $-12 \cdot 1$ \\
\hline & & $55-79$ years & 188 & 31.4 & $13 \cdot 12$ & 31.89 & 308 & $36 \cdot 2$ & 12.87 & $23 \cdot 71$ & $15 \cdot 2$ & -1.9 \\
\hline & Women & $18-34$ years & 266 & $28 \cdot 6$ & 8.69 & $17 \cdot 77$ & 280 & $25 \cdot 6$ & $6 \cdot 68$ & $15 \cdot 01$ & $-10 \cdot 5$ & $-23 \cdot 1$ \\
\hline & & $35-54$ years & 270 & $31 \cdot 1$ & 9.03 & $17 \cdot 31$ & 477 & $29 \cdot 0$ & 8.54 & $15 \cdot 42$ & -6.7 & -5.4 \\
\hline & & $55-79$ years & 196 & $26 \cdot 5$ & $7 \cdot 60$ & 14.61 & 325 & $32 \cdot 1$ & $9 \cdot 75$ & 17.55 & +21.0 & $+28 \cdot 3$ \\
\hline Croissant-like pastries, pastries, & Men & $18-34$ years & 180 & $93 \cdot 3$ & $82 \cdot 7$ & $65 \cdot 7$ & 178 & $94 \cdot 8$ & $76 \cdot 0$ & $66 \cdot 9$ & +1.5 & $-8 \cdot 2$ \\
\hline & & $35-54$ years & 245 & $89 \cdot 0$ & $70 \cdot 6$ & 67.4 & 354 & 88.7 & $62 \cdot 6$ & $52 \cdot 3$ & -0.3 & $-11 \cdot 3$ \\
\hline & & $55-79$ years & 188 & $85 \cdot 1$ & $60 \cdot 8$ & $66 \cdot 8$ & 308 & $80 \cdot 8$ & $46 \cdot 1$ & $54 \cdot 1$ & $-5 \cdot 0$ & $-24 \cdot 1^{*}$ \\
\hline & Women & $18-34$ years & 266 & $96 \cdot 6$ & $75 \cdot 1$ & $56 \cdot 7$ & 280 & 98.4 & $67 \cdot 1$ & $44 \cdot 2$ & $+1 \cdot 8$ & -10.7 \\
\hline & & $35-54$ years & 270 & 91.9 & $64 \cdot 0$ & $57 \cdot 6$ & 477 & 94.9 & $60 \cdot 1$ & $39 \cdot 3$ & +3.3 & $-6 \cdot 1$ \\
\hline & & $55-79$ years & 196 & $85 \cdot 2$ & $55 \cdot 3$ & $54 \cdot 2$ & 325 & $87 \cdot 3$ & 49.9 & $45 \cdot 4$ & +2.5 & -9.7 \\
\hline Ice cream and frozen desserts & Men & $18-34$ years & 180 & $35 \cdot 0$ & 6.88 & 12.51 & 178 & $23 \cdot 1$ & $8 \cdot 14$ & $23 \cdot 22$ & $-34 \cdot 0^{*}$ & $+18 \cdot 3$ \\
\hline & & $35-54$ years & 245 & $30 \cdot 6$ & $7 \cdot 18$ & $15 \cdot 42$ & 354 & 33.2 & $10 \cdot 45$ & $25 \cdot 31$ & +8.6 & +45.6 \\
\hline & & $55-79$ years & 188 & $25 \cdot 0$ & 5.32 & 12.95 & 308 & $28 \cdot 2$ & 8.25 & $20 \cdot 79$ & $+12 \cdot 7$ & +55.0 \\
\hline & Women & $18-34$ years & 266 & 41.4 & $9 \cdot 39$ & $16 \cdot 85$ & 280 & $41 \cdot 8$ & $9 \cdot 97$ & $17 \cdot 40$ & $+1 \cdot 0$ & $+6 \cdot 2$ \\
\hline & & $35-54$ years & 270 & $33 \cdot 7$ & 6.56 & $14 \cdot 21$ & 477 & $40 \cdot 6$ & $9 \cdot 40$ & 15.95 & +20.5 & $+43.4^{*}$ \\
\hline & & $55-79$ years & 196 & $17 \cdot 3$ & $3 \cdot 12$ & 8.71 & 325 & 29.6 & 5.64 & $11 \cdot 11$ & $+70 \cdot 8^{\star \star}$ & $+80 \cdot 8^{\star}$ \\
\hline Chocolate & Men & $18-34$ years & 180 & $40 \cdot 0$ & 6.89 & 14.90 & 178 & $59 \cdot 2$ & $12 \cdot 21$ & 23.06 & $+48 \cdot 1^{\star \star \star}$ & $+77 \cdot 2^{*}$ \\
\hline & & $35-54$ years & 245 & $28 \cdot 2$ & 3.33 & $9 \cdot 86$ & 354 & 44.4 & $5 \cdot 28$ & $12 \cdot 76$ & $+57 \cdot 8^{\star \star \star}$ & +58.4 \\
\hline & & $55-79$ years & 188 & $25 \cdot 5$ & 1.65 & 3.75 & 308 & 33.6 & 2.99 & 9.72 & +31.5 & $+81 \cdot 3^{\star}$ \\
\hline & Women & $18-34$ years & 266 & $50 \cdot 4$ & $5 \cdot 40$ & $9 \cdot 24$ & 280 & $65 \cdot 4$ & 8.45 & $11 \cdot 81$ & $+29 \cdot 9^{\star *}$ & $+56 \cdot 6^{\star \star}$ \\
\hline
\end{tabular}


WS British Journal of Nutrition

Table 3. Continued

\begin{tabular}{|c|c|c|c|c|c|c|c|c|c|c|c|c|}
\hline \multirow[b]{2}{*}{ Food group } & \multirow[b]{2}{*}{ Sex } & \multirow[b]{2}{*}{ Age } & \multicolumn{4}{|c|}{ INCA1 (1998-99) } & \multicolumn{4}{|c|}{ INCA2 (2006-07) } & \multicolumn{2}{|c|}{ Trends } \\
\hline & & & $n$ & $\%$ & Mean & SD & $n$ & $\%$ & Mean & SD & $\%$ & Mean (\%) \\
\hline & & $35-54$ years & 270 & $34 \cdot 8$ & $2 \cdot 86$ & $7 \cdot 68$ & 477 & $56 \cdot 2$ & 5.32 & 8.60 & $+61 \cdot 4^{\star \star \star}$ & $+86 \cdot 2^{\star * \star}$ \\
\hline & & $55-79$ years & 196 & $29 \cdot 1$ & $2 \cdot 24$ & 7.79 & 325 & $42 \cdot 8$ & 3.15 & $7 \cdot 82$ & $+47 \cdot 0^{\star \star}$ & +40.8 \\
\hline \multirow[t]{6}{*}{ Sugar and confectionery } & Men & $18-34$ years & 180 & $87 \cdot 8$ & 24.9 & $27 \cdot 7$ & 178 & 82.7 & $14 \cdot 8$ & $22 \cdot 7$ & -5.8 & $-40 \cdot 3^{\star \star \star}$ \\
\hline & & $35-54$ years & 245 & $94 \cdot 3$ & 31.9 & $29 \cdot 3$ & 354 & 90.1 & $23 \cdot 1$ & $20 \cdot 7$ & $-4 \cdot 4$ & $-27 \cdot 7^{\star \star \star}$ \\
\hline & & $55-79$ years & 188 & $93 \cdot 1$ & 30.8 & 24.4 & 308 & 81.9 & $25 \cdot 0$ & $28 \cdot 7$ & $-12 \cdot 0^{\star \star \star}$ & $-19 \cdot 0^{*}$ \\
\hline & Women & $18-34$ years & 266 & $88 \cdot 3$ & $22 \cdot 1$ & $22 \cdot 1$ & 280 & 87.6 & $16 \cdot 9$ & $17 \cdot 7$ & -0.9 & $-23 \cdot 2^{\star \star}$ \\
\hline & & $35-54$ years & 270 & $93 \cdot 0$ & $27 \cdot 6$ & $23 \cdot 6$ & 477 & 91.3 & $21 \cdot 1$ & $19 \cdot 1$ & $-1 \cdot 8$ & $-23 \cdot 7^{\star \star *}$ \\
\hline & & $55-79$ years & 196 & 96.9 & $28 \cdot 2$ & $19 \cdot 7$ & 325 & 85.9 & $22 \cdot 4$ & $19 \cdot 1$ & $-11 \cdot 4^{\star \star \star}$ & $-20 \cdot 6^{\star \star}$ \\
\hline \multirow[t]{6}{*}{ Milk } & Men & $18-34$ years & 180 & $72 \cdot 2$ & 129.6 & $135 \cdot 3$ & 178 & 56.9 & $121 \cdot 2$ & $185 \cdot 2$ & $-21 \cdot 3^{\star \star}$ & -6.4 \\
\hline & & $35-54$ years & 245 & 60.0 & 93.8 & $113 \cdot 1$ & 354 & $46 \cdot 9$ & $89 \cdot 3$ & $197 \cdot 8$ & $-21 \cdot 8^{\star \star}$ & $-4 \cdot 8$ \\
\hline & & $55-79$ years & 188 & $60 \cdot 6$ & $100 \cdot 5$ & $125 \cdot 2$ & 308 & 44.5 & 72.5 & $135 \cdot 5$ & $-26 \cdot 7^{\star \star \star}$ & $-27 \cdot 9^{\star}$ \\
\hline & Women & $18-34$ years & 266 & $82 \cdot 0$ & $152 \cdot 6$ & $128 \cdot 2$ & 280 & 65.0 & $102 \cdot 8$ & $134 \cdot 1$ & $-20 \cdot 7^{\star \star \star}$ & $-32 \cdot 6^{\star \star *}$ \\
\hline & & $35-54$ years & 270 & $64 \cdot 1$ & $108 \cdot 2$ & 124.7 & 477 & $49 \cdot 3$ & 84.9 & $116 \cdot 8$ & $-23 \cdot 0^{\star \star \star}$ & $-21 \cdot 5^{\star}$ \\
\hline & & $55-79$ years & 196 & $65 \cdot 3$ & 118.9 & 137.5 & 325 & 47.8 & 74.5 & $111 \cdot 3$ & $-26 \cdot 8^{\star \star \star}$ & $-37 \cdot 4^{\star \star *}$ \\
\hline \multirow[t]{6}{*}{ Cheese } & Men & $18-34$ years & 180 & $87 \cdot 2$ & $37 \cdot 7$ & $34 \cdot 2$ & 178 & $87 \cdot 3$ & $33 \cdot 2$ & 39.2 & +0.1 & -11.9 \\
\hline & & $35-54$ years & 245 & $97 \cdot 1$ & 50.9 & 34.9 & 354 & $92 \cdot 8$ & 38.7 & 28.5 & $-4 \cdot 5^{\star}$ & $-23 \cdot 8^{\star \star \star}$ \\
\hline & & $55-79$ years & 188 & $96 \cdot 8$ & $52 \cdot 8$ & $42 \cdot 0$ & 308 & $96 \cdot 3$ & $47 \cdot 2$ & 38.5 & -0.5 & $-10 \cdot 6$ \\
\hline & Women & $18-34$ years & 266 & 92.5 & $29 \cdot 1$ & $22 \cdot 9$ & 280 & 90.1 & $26 \cdot 2$ & $25 \cdot 8$ & -2.5 & -9.7 \\
\hline & & $35-54$ years & 270 & 94.4 & 35.0 & 25.4 & 477 & 93.8 & $28 \cdot 3$ & 19.4 & -0.6 & $-19 \cdot 1^{\star \star \star}$ \\
\hline & & $55-79$ years & 196 & 95.4 & 37.3 & 25.4 & 325 & 94.3 & 28.0 & $19 \cdot 3$ & -1.2 & $-24 \cdot 9^{* \star *}$ \\
\hline \multirow{6}{*}{ Other dairy products } & Men & $18-34$ years & 180 & $86 \cdot 1$ & 88.5 & 84.0 & 178 & 83.6 & 84.5 & $110 \cdot 3$ & -2.9 & -4.5 \\
\hline & & $35-54$ years & 245 & $75 \cdot 5$ & 58.2 & 74.7 & 354 & 79.9 & 79.6 & $90 \cdot 2$ & $+5 \cdot 8$ & $+36 \cdot 7^{\star \star}$ \\
\hline & & $55-79$ years & 188 & 66.5 & 54.6 & 68.9 & 308 & 73.1 & $65 \cdot 2$ & $91 \cdot 2$ & +9.9 & +19.5 \\
\hline & Women & $18-34$ years & 266 & $89 \cdot 1$ & 80.7 & 69.8 & 280 & 89.2 & 71.4 & $68 \cdot 0$ & +0.1 & -11.5 \\
\hline & & $35-54$ years & 270 & 87.0 & 83.6 & $76 \cdot 4$ & 477 & 93.3 & $103 \cdot 8$ & 75.4 & $+7 \cdot 2^{\star}$ & $+24 \cdot 3^{\star \star}$ \\
\hline & & $55-79$ years & 196 & $85 \cdot 2$ & 89.9 & $83 \cdot 8$ & 325 & 86.0 & $93 \cdot 6$ & $65 \cdot 8$ & +0.9 & $+4 \cdot 1$ \\
\hline \multirow[t]{6}{*}{ Cream desserts } & Men & $18-34$ years & 180 & $62 \cdot 8$ & $36 \cdot 6$ & $46 \cdot 1$ & 178 & 61.3 & $30 \cdot 8$ & $51 \cdot 7$ & $-2 \cdot 4$ & $-15 \cdot 9$ \\
\hline & & $35-54$ years & 245 & $48 \cdot 2$ & 24.8 & 37.0 & 354 & 54.0 & 30.4 & 44.0 & $+12 \cdot 1$ & +22.6 \\
\hline & & $55-79$ years & 188 & $37 \cdot 8$ & $19 \cdot 4$ & $35 \cdot 2$ & 308 & 41.0 & $20 \cdot 4$ & $40 \cdot 3$ & $+8 \cdot 7$ & +4.8 \\
\hline & Women & $18-34$ years & 266 & $56 \cdot 8$ & $26 \cdot 0$ & 33.5 & 280 & $57 \cdot 0$ & $22 \cdot 8$ & $33 \cdot 8$ & +0.4 & $-12 \cdot 5$ \\
\hline & & $35-54$ years & 270 & $51 \cdot 1$ & $19 \cdot 7$ & $28 \cdot 8$ & 477 & $57 \cdot 2$ & $24 \cdot 7$ & 29.8 & +11.8 & +25.6 \\
\hline & & $55-79$ years & 196 & $40 \cdot 3$ & $18 \cdot 3$ & $30 \cdot 3$ & 325 & 53.6 & 29.6 & $52 \cdot 1$ & $+33 \cdot 1^{\star \star}$ & $+61 \cdot 3^{\star}$ \\
\hline \multirow[t]{6}{*}{ Butter } & Men & $18-34$ years & 180 & $100 \cdot 0$ & $14 \cdot 1$ & 11.5 & 178 & 80.6 & 9.7 & $14 \cdot 1$ & ND & ND \\
\hline & & $35-54$ years & 245 & $100 \cdot 0$ & $15 \cdot 0$ & 11.4 & 354 & 83.6 & $12 \cdot 1$ & $13 \cdot 6$ & ND & ND \\
\hline & & $55-79$ years & 188 & 99.5 & $15 \cdot 5$ & $12 \cdot 1$ & 308 & $82 \cdot 3$ & $13 \cdot 0$ & $14 \cdot 7$ & ND & ND \\
\hline & Women & $18-34$ years & 266 & $100 \cdot 0$ & 11.7 & $9 \cdot 2$ & 280 & 83.1 & 8.8 & 9.5 & ND & ND \\
\hline & & $35-54$ years & 270 & $100 \cdot 0$ & 14.2 & $10 \cdot 4$ & 477 & 85.5 & $10 \cdot 6$ & $9 \cdot 0$ & ND & ND \\
\hline & & $55-79$ years & 196 & 99.0 & $12 \cdot 9$ & 9.5 & 325 & 82.4 & 11.4 & $10 \cdot 4$ & ND & ND \\
\hline \multirow[t]{6}{*}{ Oil } & Men & $18-34$ years & 180 & $90 \cdot 0$ & 1.9 & 2.4 & 178 & 70.0 & $6 \cdot 3$ & 9.7 & ND & ND \\
\hline & & $35-54$ years & 245 & 93.5 & $2 \cdot 3$ & 4.3 & 354 & 83.7 & $10 \cdot 1$ & 9.7 & ND & ND \\
\hline & & $55-79$ years & 188 & $95 \cdot 2$ & $2 \cdot 6$ & 4.8 & 308 & 85.9 & 12.5 & $13 \cdot 2$ & ND & ND \\
\hline & Women & $18-34$ years & 266 & $87 \cdot 2$ & 1.7 & 2.4 & 280 & $85 \cdot 2$ & 9.8 & $9 \cdot 2$ & ND & ND \\
\hline & & $35-54$ years & 270 & $92 \cdot 6$ & $2 \cdot 1$ & 3.5 & 477 & 87.8 & $11 \cdot 2$ & 8.6 & ND & ND \\
\hline & & $55-79$ years & 196 & 94.4 & $2 \cdot 8$ & $5 \cdot 8$ & 325 & 91.5 & $13 \cdot 0$ & 9.8 & ND & ND \\
\hline \multirow{6}{*}{ Margarines } & Men & $18-34$ years & 180 & 99.4 & 3.8 & 4.4 & 178 & $27 \cdot 4$ & $2 \cdot 3$ & 5.9 & ND & ND \\
\hline & & $35-54$ years & 245 & $99 \cdot 2$ & 3.6 & $2 \cdot 7$ & 354 & 33.8 & 3.8 & $7 \cdot 7$ & ND & ND \\
\hline & & $55-79$ years & 188 & 98.4 & 5.0 & $6 \cdot 3$ & 308 & $47 \cdot 2$ & 6.6 & 11.5 & ND & ND \\
\hline & Women & $18-34$ years & 266 & 98.9 & $2 \cdot 7$ & 1.9 & 280 & 36.0 & $2 \cdot 4$ & 4.6 & ND & ND \\
\hline & & $35-54$ years & 270 & $99 \cdot 3$ & 3.5 & 3.4 & 477 & 44.1 & 3.9 & $6 \cdot 2$ & ND & ND \\
\hline & & $55-79$ years & 196 & 95.4 & 4.0 & 5.2 & 325 & 50.0 & 6.7 & 9.6 & ND & ND \\
\hline
\end{tabular}


WS British Journal of Nutrition

Table 3. Continued

\begin{tabular}{|c|c|c|c|c|c|c|c|c|c|c|c|c|}
\hline \multirow[b]{2}{*}{ Food group } & \multirow[b]{2}{*}{ Sex } & \multirow[b]{2}{*}{ Age } & \multicolumn{4}{|c|}{ INCA1 (1998-99) } & \multicolumn{4}{|c|}{ INCA2 (2006-07) } & \multicolumn{2}{|c|}{ Trends } \\
\hline & & & $n$ & $\%$ & Mean & SD & $n$ & $\%$ & Mean & SD & $\%$ & Mean (\%) \\
\hline \multirow[t]{6}{*}{ Other fats } & Men & $18-34$ years & 180 & I & I & I & 178 & 1.9 & 0.1 & $1 \cdot 1$ & ND & ND \\
\hline & & $35-54$ years & 245 & I & I & I & 354 & $2 \cdot 4$ & 0.1 & 0.8 & ND & ND \\
\hline & & $55-79$ years & 188 & $1 \cdot 1$ & 0.0 & 0.3 & 308 & $4 \cdot 1$ & 0.2 & 1.7 & ND & ND \\
\hline & Women & $18-34$ years & 266 & 0.8 & 0.1 & 0.8 & 280 & 0.8 & 0.0 & 0.2 & ND & ND \\
\hline & & $35-54$ years & 270 & 1 & / & I & 477 & $2 \cdot 6$ & 0.1 & 0.6 & ND & ND \\
\hline & & $55-79$ years & 196 & 1.5 & 0.0 & 0.4 & 325 & 4.5 & 0.2 & 0.9 & ND & ND \\
\hline \multirow{6}{*}{ Eggs and egg products } & Men & $18-34$ years & 180 & $61 \cdot 1$ & $19 \cdot 3$ & $26 \cdot 8$ & 178 & $47 \cdot 8$ & $13 \cdot 8$ & $24 \cdot 2$ & $-21 \cdot 8^{\star}$ & $-28 \cdot 4^{\star}$ \\
\hline & & $35-54$ years & 245 & $68 \cdot 6$ & $20 \cdot 1$ & $24 \cdot 7$ & 354 & 57.6 & 14.2 & $16 \cdot 8$ & $-16 \cdot 0^{\star}$ & $-29.4^{\star \star}$ \\
\hline & & $55-79$ years & 188 & $71 \cdot 3$ & $23 \cdot 3$ & $25 \cdot 0$ & 308 & $72 \cdot 4$ & $18 \cdot 0$ & $18 \cdot 6$ & +1.5 & $-22 \cdot 8^{\star *}$ \\
\hline & Women & $18-34$ years & 266 & $58 \cdot 3$ & $13 \cdot 0$ & $16 \cdot 0$ & 280 & $56 \cdot 6$ & $12 \cdot 1$ & $17 \cdot 4$ & $-2 \cdot 8$ & -6.5 \\
\hline & & $35-54$ years & 270 & $73 \cdot 7$ & $17 \cdot 2$ & $16 \cdot 3$ & 477 & 70.9 & $14 \cdot 8$ & 13.9 & -3.8 & $-14 \cdot 3^{*}$ \\
\hline & & $55-79$ years & 196 & 69.4 & $17 \cdot 8$ & $19 \cdot 1$ & 325 & 74.7 & $16 \cdot 6$ & $15 \cdot 7$ & +7.6 & -6.7 \\
\hline \multirow{6}{*}{ Meat } & Men & $18-34$ years & 180 & 93.9 & $68 \cdot 7$ & $49 \cdot 7$ & 178 & 90.6 & $66 \cdot 7$ & $58 \cdot 3$ & -3.5 & -3.0 \\
\hline & & $35-54$ years & 245 & 97.1 & $70 \cdot 0$ & 44.0 & 354 & 92.9 & 63.4 & $44 \cdot 7$ & $-4 \cdot 3^{*}$ & -9.4 \\
\hline & & $55-79$ years & 188 & $96 \cdot 8$ & $58 \cdot 3$ & 41.5 & 308 & $96 \cdot 1$ & $55 \cdot 1$ & $40 \cdot 8$ & -0.7 & $-5 \cdot 6$ \\
\hline & Women & $18-34$ years & 266 & 91.4 & $47 \cdot 1$ & $33 \cdot 1$ & 280 & 87.6 & $39 \cdot 3$ & $28 \cdot 2$ & $-4 \cdot 1$ & $-16 \cdot 4^{\star \star}$ \\
\hline & & $35-54$ years & 270 & $95 \cdot 2$ & $53 \cdot 1$ & 33.9 & 477 & 91.6 & $40 \cdot 7$ & $26 \cdot 5$ & -3.7 & $-23 \cdot 4^{* * *}$ \\
\hline & & $55-79$ years & 196 & $91 \cdot 8$ & 43.5 & $29 \cdot 4$ & 325 & 90.4 & $38 \cdot 2$ & $25 \cdot 1$ & -1.5 & $-12 \cdot 2^{\star}$ \\
\hline \multirow[t]{6}{*}{ Poultry and game } & Men & $18-34$ years & 180 & $78 \cdot 3$ & $36 \cdot 2$ & 34.9 & 178 & $74 \cdot 3$ & 41.4 & $62 \cdot 2$ & $-5 \cdot 2$ & $+14 \cdot 2$ \\
\hline & & $35-54$ years & 245 & $81 \cdot 6$ & $39 \cdot 3$ & 39.9 & 354 & 79.2 & $41 \cdot 1$ & $43 \cdot 6$ & -3.0 & +4.6 \\
\hline & & $55-79$ years & 188 & $79 \cdot 3$ & 35.8 & $32 \cdot 6$ & 308 & $75 \cdot 6$ & $36 \cdot 7$ & $40 \cdot 1$ & -4.6 & +2.5 \\
\hline & Women & $18-34$ years & 266 & $80 \cdot 8$ & $28 \cdot 3$ & $27 \cdot 0$ & 280 & $74 \cdot 6$ & $23 \cdot 6$ & $25 \cdot 2$ & -7.7 & $-16 \cdot 5^{\star}$ \\
\hline & & $35-54$ years & 270 & $80 \cdot 4$ & 30.5 & $30 \cdot 2$ & 477 & 74.8 & $26 \cdot 8$ & $27 \cdot 1$ & $-6 \cdot 9$ & $-12 \cdot 2$ \\
\hline & & $55-79$ years & 196 & $75 \cdot 0$ & 29.8 & 29.1 & 325 & 71.8 & $24 \cdot 1$ & 28.0 & $-4 \cdot 3$ & $-19 \cdot 0^{*}$ \\
\hline \multirow{6}{*}{ Offal } & Men & $18-34$ years & 180 & $13 \cdot 3$ & 2.91 & 8.61 & 178 & $11 \cdot 8$ & 1.77 & 7.03 & $-11 \cdot 3$ & -39.0 \\
\hline & & $35-54$ years & 245 & $18 \cdot 8$ & 3.63 & 8.83 & 354 & 14.0 & 2.64 & 7.48 & $-25 \cdot 5$ & $-27 \cdot 1$ \\
\hline & & $55-79$ years & 188 & $18 \cdot 6$ & 3.84 & 9.90 & 308 & $21 \cdot 7$ & 4.77 & 12.05 & $+16 \cdot 5$ & +24.0 \\
\hline & Women & $18-34$ years & 266 & 13.9 & 2.48 & 6.75 & 280 & $10 \cdot 6$ & 1.58 & 5.02 & -23.8 & -36.5 \\
\hline & & $35-54$ years & 270 & $16 \cdot 3$ & 2.63 & 7.02 & 477 & $18 \cdot 0$ & $3 \cdot 10$ & 6.88 & $+10 \cdot 2$ & +18.0 \\
\hline & & $55-79$ years & 196 & 20.9 & 3.95 & $10 \cdot 26$ & 325 & $21 \cdot 0$ & 3.44 & $7 \cdot 24$ & +0.4 & $-12 \cdot 9$ \\
\hline \multirow[t]{6}{*}{ Meat products } & Men & $18-34$ years & 180 & 93.9 & $47 \cdot 8$ & $42 \cdot 2$ & 178 & $87 \cdot 1$ & 41.0 & 41.3 & $-7 \cdot 2^{\star}$ & -14.1 \\
\hline & & $35-54$ years & 245 & $96 \cdot 3$ & $47 \cdot 2$ & 31.5 & 354 & 93.8 & $42 \cdot 9$ & 33.4 & $-2 \cdot 7$ & $-9 \cdot 1$ \\
\hline & & $55-79$ years & 188 & $92 \cdot 0$ & $39 \cdot 1$ & $35 \cdot 2$ & 308 & 95.1 & $41 \cdot 7$ & $36 \cdot 3$ & +3.4 & +6.6 \\
\hline & Women & $18-34$ years & 266 & $90 \cdot 6$ & $32 \cdot 7$ & 28.4 & 280 & 87.5 & $26 \cdot 8$ & $21 \cdot 8$ & -3.4 & $-18 \cdot 3^{\star \star}$ \\
\hline & & $35-54$ years & 270 & $92 \cdot 2$ & $27 \cdot 8$ & $22 \cdot 8$ & 477 & 92.4 & $30 \cdot 0$ & 20.5 & +0.2 & $+7 \cdot 6$ \\
\hline & & $55-79$ years & 196 & $90 \cdot 8$ & $27 \cdot 1$ & $27 \cdot 2$ & 325 & 89.2 & $27 \cdot 2$ & $20 \cdot 1$ & -1.8 & +0.5 \\
\hline \multirow[t]{6}{*}{ Fish } & Men & $18-34$ years & 180 & 74.4 & $22 \cdot 1$ & $21 \cdot 3$ & 178 & $66 \cdot 2$ & $18 \cdot \overline{8}$ & 27.5 & $-11 \cdot 1$ & -14.6 \\
\hline & & $35-54$ years & 245 & $82 \cdot 0$ & $28 \cdot 7$ & $31 \cdot 7$ & 354 & $75 \cdot 1$ & $24 \cdot 7$ & 24.1 & -8.4 & -13.9 \\
\hline & & $55-79$ years & 188 & 80.9 & 31.3 & $30 \cdot 4$ & 308 & $84 \cdot 1$ & 31.5 & $30 \cdot 2$ & +4.0 & +0.8 \\
\hline & Women & $18-34$ years & 266 & $74 \cdot 8$ & $21 \cdot 1$ & $21 \cdot 6$ & 280 & 70.9 & $20 \cdot 1$ & 20.5 & -5.3 & -5.0 \\
\hline & & $35-54$ years & 270 & 81.9 & $25 \cdot 4$ & 23.7 & 477 & 83.6 & $27 \cdot 8$ & 22.4 & $+2 \cdot 2$ & +9.5 \\
\hline & & $55-79$ years & 196 & $84 \cdot 2$ & 28.9 & $27 \cdot 6$ & 325 & 92.8 & $31 \cdot 2$ & $22 \cdot 9$ & $+10 \cdot 2^{* *}$ & +7.8 \\
\hline \multirow[t]{6}{*}{ Crustaceans and molluscs } & Men & $18-34$ years & 180 & $21 \cdot 1$ & 2.96 & 7.02 & 178 & 24.0 & $2 \cdot 37$ & 7.39 & +13.8 & -19.9 \\
\hline & & $35-54$ years & 245 & $31 \cdot 0$ & 5.32 & $11 \cdot 12$ & 354 & 34.7 & $5 \cdot 16$ & $10 \cdot 38$ & +11.8 & -3.0 \\
\hline & & $55-79$ years & 188 & 33.5 & 5.79 & 11.43 & 308 & 34.0 & $5 \cdot 11$ & $10 \cdot 90$ & +1.4 & -11.8 \\
\hline & Women & $18-34$ years & 266 & 29.7 & 4.60 & 11.41 & 280 & 34.0 & $4 \cdot 31$ & $10 \cdot 25$ & +14.4 & $-6 \cdot 2$ \\
\hline & & $35-54$ years & 270 & $29 \cdot 3$ & 4.47 & 10.03 & 477 & $35 \cdot 1$ & 4.57 & 8.02 & +20.0 & $+2 \cdot 3$ \\
\hline & & $55-79$ years & 196 & $29 \cdot 1$ & 4.61 & 10.73 & 325 & $36 \cdot 8$ & 4.74 & 8.96 & +26.6 & $+2 \cdot 8$ \\
\hline \multirow[t]{2}{*}{ Vegetables } & Men & $18-34$ years & 180 & $96 \cdot 1$ & $109 \cdot 7$ & 65.9 & 178 & $96 \cdot 2$ & 94.6 & $86 \cdot 8$ & +0.1 & $-13 \cdot 8^{\star}$ \\
\hline & & $35-54$ years & 245 & 98.4 & $137 \cdot 3$ & $76 \cdot 7$ & 354 & 98.8 & 137.8 & 84.7 & +0.4 & +0.4 \\
\hline
\end{tabular}


WS British Journal of Nutrition

Table 3. Continued

\begin{tabular}{|c|c|c|c|c|c|c|c|c|c|c|c|c|}
\hline \multirow[b]{2}{*}{ Food group } & \multirow[b]{2}{*}{ Sex } & \multirow[b]{2}{*}{ Age } & \multicolumn{4}{|c|}{ INCA1 (1998-99) } & \multicolumn{4}{|c|}{ INCA2 (2006-07) } & \multicolumn{2}{|c|}{ Trends } \\
\hline & & & $n$ & $\%$ & Mean & SD & $n$ & $\%$ & Mean & SD & $\%$ & Mean (\%) \\
\hline & & $55-79$ years & 188 & 98.9 & 147.4 & $81 \cdot 6$ & 308 & 99.5 & $159 \cdot 4$ & 98.0 & +0.6 & +8.2 \\
\hline & Women & $18-34$ years & 266 & 98.1 & $112 \cdot 9$ & $70 \cdot 2$ & 280 & 98.8 & 114.0 & $82 \cdot 2$ & +0.7 & +1.0 \\
\hline & & $35-54$ years & 270 & 99.3 & 140.5 & 71.4 & 477 & 99.7 & 144.9 & 67.5 & +0.4 & $+3 \cdot 1$ \\
\hline \multirow{7}{*}{ Fruit } & & $55-79$ years & 196 & 98.5 & 143.4 & 89.2 & 325 & 99.2 & 164.9 & $76 \cdot 7$ & +0.7 & $+15 \cdot 0^{\star}$ \\
\hline & Men & $18-34$ years & 180 & 73.3 & 72.6 & $82 \cdot 6$ & 178 & $70 \cdot 4$ & $72 \cdot 8$ & 111.3 & $-4 \cdot 1$ & +0.3 \\
\hline & & $35-54$ years & 245 & 79.6 & $100 \cdot 8$ & $105 \cdot 2$ & 354 & 83.1 & 138.9 & 164.5 & +4.4 & $+37 \cdot 8^{\star *}$ \\
\hline & & $55-79$ years & 188 & 90.4 & 176.6 & 154.3 & 308 & 91.3 & $185 \cdot 2$ & $166 \cdot 2$ & +1.0 & +4.8 \\
\hline & Women & $18-34$ years & 266 & 81.2 & $77 \cdot 7$ & 81.5 & 280 & 84.0 & $75 \cdot 1$ & 71.6 & +3.5 & -3.3 \\
\hline & & $35-54$ years & 270 & 87.0 & $108 \cdot 1$ & 99.5 & 477 & 93.5 & 160.5 & 123.6 & $+7 \cdot 4^{\star \star}$ & $+48 \cdot 4^{\star \star \star}$ \\
\hline & & $55-79$ years & 196 & 96.9 & 163.6 & 116.8 & 325 & 97.8 & 201.8 & 136.9 & +0.8 & $+23 \cdot 4^{\star \star}$ \\
\hline \multirow[t]{6}{*}{ Mashed and cooked fruit } & Men & $18-34$ years & 180 & $22 \cdot 2$ & 8.71 & $20 \cdot 27$ & 178 & $20 \cdot 4$ & 7.43 & 24.48 & -8.2 & $-14 \cdot 7$ \\
\hline & & $35-54$ years & 245 & 24.5 & 8.51 & 20.53 & 354 & 27.9 & $9 \cdot 19$ & 19.67 & +14.0 & +8.0 \\
\hline & & $55-79$ years & 188 & 30.9 & 13.01 & 27.00 & 308 & 27.6 & $11 \cdot 21$ & $25 \cdot 79$ & $-10 \cdot 6$ & -13.9 \\
\hline & Women & $18-34$ years & 266 & $31 \cdot 2$ & 11.08 & 21.79 & 280 & 35.9 & $9 \cdot 42$ & $18 \cdot 18$ & $+15 \cdot 0$ & $-15 \cdot 0$ \\
\hline & & $35-54$ years & 270 & 27.8 & $9 \cdot 12$ & 19.08 & 477 & 40.9 & 16.48 & $26 \cdot 73$ & $+47 \cdot 3^{\star \star}$ & $+80 \cdot 6^{\star \star \star}$ \\
\hline & & $55-79$ years & 196 & 34.7 & $16 \cdot 12$ & 35.73 & 325 & $47 \cdot 7$ & 25.42 & 52.09 & $+37.5^{\star \star}$ & +57.7 \\
\hline \multirow[t]{6}{*}{ Dried fruit, nuts and seeds } & Men & $18-34$ years & 180 & $18 \cdot 3$ & 1.34 & 4.30 & 178 & 23.5 & 1.95 & $7 \cdot 60$ & +28.4 & +44.9 \\
\hline & & $35-54$ years & 245 & 23.3 & $2 \cdot 80$ & $9 \cdot 17$ & 354 & $45 \cdot 0$ & 3.75 & 7.41 & $+93 \cdot 4^{\star \star \star}$ & +33.7 \\
\hline & & $55-79$ years & 188 & 23.4 & $2 \cdot 89$ & 9.06 & 308 & 33.0 & 3.28 & $9 \cdot 31$ & $+41 \cdot 1^{*}$ & +13.4 \\
\hline & Women & $18-34$ years & 266 & 18.4 & 1.26 & $4 \cdot 76$ & 280 & $20 \cdot 8$ & 1.01 & 3.46 & $+13 \cdot 2$ & $-19 \cdot 4$ \\
\hline & & $35-54$ years & 270 & $25 \cdot 6$ & 1.52 & 3.64 & 477 & 37.5 & 3.23 & $6 \cdot 37$ & $+46 \cdot 7^{\star \star}$ & $+112 \cdot 5^{\star \star \star}$ \\
\hline & & $55-79$ years & 196 & 23.0 & $4 \cdot 20$ & $15 \cdot 21$ & 325 & 29.9 & 2.83 & $6 \cdot 71$ & +30.0 & -32.7 \\
\hline \multirow[t]{6}{*}{ Breakfast cereals } & Men & $18-34$ years & 180 & $18 \cdot 3$ & 5.25 & 15.65 & 178 & 23.4 & $7 \cdot 31$ & $22 \cdot 22$ & +27.7 & +39.1 \\
\hline & & $35-54$ years & 245 & 9.4 & 2.05 & 9.71 & 354 & $12 \cdot 0$ & $3 \cdot 16$ & 12.78 & +27.6 & +54.7 \\
\hline & & $55-79$ years & 188 & 7.4 & 2.65 & $11 \cdot 21$ & 308 & 7.9 & 4.09 & $22 \cdot 70$ & +5.5 & +54.2 \\
\hline & Women & $18-34$ years & 266 & $24 \cdot 1$ & $7 \cdot 24$ & 17.71 & 280 & 35.5 & $8 \cdot 10$ & 17.61 & $+47 \cdot 6^{\star *}$ & +11.9 \\
\hline & & $35-54$ years & 270 & $15 \cdot 9$ & 4.73 & 14.07 & 477 & 19.7 & $5 \cdot 16$ & 12.77 & +23.6 & +9.2 \\
\hline & & $55-79$ years & 196 & $9 \cdot 2$ & 2.08 & 9.03 & 325 & $10 \cdot 2$ & 3.26 & 12.47 & $+10 \cdot 8$ & +56.8 \\
\hline \multirow[t]{6}{*}{ Pizza, savoury pastries } & Men & $18-34$ years & 180 & 68.3 & $41 \cdot 1$ & 44.7 & 178 & 68.9 & 49.9 & 73.9 & +0.8 & +21.5 \\
\hline & & $35-54$ years & 245 & $58 \cdot 8$ & 23.5 & 30.5 & 354 & $58 \cdot 2$ & 31.2 & 37.9 & -1.0 & $+33.0^{\star}$ \\
\hline & & $55-79$ years & 188 & $42 \cdot 0$ & $14 \cdot 2$ & 23.4 & 308 & 38.6 & $14 \cdot 1$ & $25 \cdot 2$ & -8.3 & -0.7 \\
\hline & Women & $18-34$ years & 266 & 68.0 & $28 \cdot 3$ & 35.2 & 280 & $68 \cdot 3$ & $29 \cdot 1$ & $30 \cdot 1$ & +0.4 & +2.6 \\
\hline & & $35-54$ years & 270 & $61 \cdot 1$ & $19 \cdot 3$ & $27 \cdot 3$ & 477 & 55.1 & $17 \cdot 7$ & $21 \cdot 3$ & -9.9 & -8.3 \\
\hline & & $55-79$ years & 196 & 38.8 & 11.7 & $25 \cdot 9$ & 325 & $41 \cdot 0$ & 11.8 & $18 \cdot 1$ & $+5 \cdot 7$ & +0.7 \\
\hline \multirow[t]{6}{*}{ Sandwiches and hamburgers } & Men & $18-34$ years & 180 & 60.6 & $42 \cdot 3$ & $52 \cdot 1$ & 178 & $62 \cdot 2$ & $44 \cdot 8$ & $67 \cdot 2$ & $+2 \cdot 8$ & +5.9 \\
\hline & & $35-54$ years & 245 & $44 \cdot 1$ & $19 \cdot 2$ & $32 \cdot 1$ & 354 & 43.9 & $22 \cdot 6$ & $41 \cdot 1$ & -0.3 & +17.9 \\
\hline & & $55-79$ years & 188 & $19 \cdot 7$ & $5 \cdot 6$ & 14.0 & 308 & $17 \cdot 3$ & 4.5 & $16 \cdot 0$ & $-12 \cdot 1$ & $-19 \cdot 2$ \\
\hline & Women & $18-34$ years & 266 & $50 \cdot 8$ & $18 \cdot 0$ & 24.9 & 280 & 58.0 & 22.5 & $35 \cdot 8$ & $+14 \cdot 2$ & +24.9 \\
\hline & & $35-54$ years & 270 & 35.2 & 12.0 & 22.2 & 477 & 34.0 & 9.9 & $16 \cdot 5$ & $-3 \cdot 3$ & -17.7 \\
\hline & & $55-79$ years & 196 & $17 \cdot 9$ & 3.9 & $12 \cdot 1$ & 325 & $18 \cdot 6$ & 4.7 & $12 \cdot 6$ & +4.3 & $+18 \cdot 8$ \\
\hline \multirow[t]{6}{*}{ Soups } & Men & $18-34$ years & 180 & 37.8 & 43.3 & $73 \cdot 1$ & 178 & $25 \cdot 3$ & $31 \cdot 2$ & $80 \cdot 1$ & $-33 \cdot 1^{\star}$ & -28.0 \\
\hline & & $35-54$ years & 245 & 49.8 & 76.6 & 112.1 & 354 & 44.6 & 68.8 & 119.8 & -10.4 & -10.2 \\
\hline & & $55-79$ years & 188 & 64.9 & 143.0 & 151.5 & 308 & $61 \cdot 9$ & $128 \cdot 3$ & 174.3 & -4.6 & $-10 \cdot 3$ \\
\hline & Women & $18-34$ years & 266 & $41 \cdot 7$ & $47 \cdot 6$ & 78.8 & 280 & $47 \cdot 6$ & 51.8 & 94.8 & $+14 \cdot 2$ & +8.8 \\
\hline & & $35-54$ years & 270 & 53.7 & 78.1 & 111.4 & 477 & $51 \cdot 2$ & 68.2 & 93.6 & -4.7 & -12.6 \\
\hline & & $55-79$ years & 196 & 74.5 & 158.8 & 134.9 & 325 & 68.9 & 139.0 & 138.0 & -7.5 & -12.5 \\
\hline \multirow[t]{4}{*}{ Mixed dishes } & Men & $18-34$ years & 180 & 83.3 & $93 \cdot 2$ & $86 \cdot 3$ & 178 & $90 \cdot 7$ & $93 \cdot 0$ & $101 \cdot 6$ & $+8 \cdot 8^{*}$ & -0.3 \\
\hline & & $35-54$ years & 245 & 83.7 & 97.0 & 89.2 & 354 & 87.9 & 84.2 & 71.7 & +5.0 & $-13 \cdot 1$ \\
\hline & & $55-79$ years & 188 & $79 \cdot 3$ & $69 \cdot 7$ & $65 \cdot 0$ & 308 & 83.4 & $68 \cdot 0$ & 73.9 & $+5 \cdot 2$ & -2.4 \\
\hline & Women & $18-34$ years & 266 & 85.3 & 75.8 & 67.3 & 280 & 88.9 & 65.9 & 57.7 & $\begin{array}{l}1.2 \\
+4.2\end{array}$ & -13.0 \\
\hline
\end{tabular}


Table 3. Continued

\begin{tabular}{|c|c|c|c|c|c|c|c|c|c|c|c|c|}
\hline \multirow[b]{2}{*}{ Food group } & \multirow[b]{2}{*}{ Sex } & \multirow[b]{2}{*}{ Age } & \multicolumn{4}{|c|}{ INCA1 (1998-99) } & \multicolumn{4}{|c|}{ INCA2 (2006-07) } & \multicolumn{2}{|c|}{ Trends } \\
\hline & & & $n$ & $\%$ & Mean & SD & $n$ & $\%$ & Mean & SD & $\%$ & Mean (\%) \\
\hline & & $35-54$ years & 270 & $84 \cdot 8$ & 64.4 & $61 \cdot 0$ & 477 & 87.9 & $64 \cdot 2$ & 49.6 & +3.7 & -0.3 \\
\hline & & $55-79$ years & 196 & 75.5 & 51.7 & 55.2 & 325 & $79 \cdot 3$ & 54.0 & 53.1 & +5.0 & +4.5 \\
\hline \multirow{6}{*}{ Waters } & Men & $18-34$ years & 180 & 93.9 & $584 \cdot 8$ & 417.8 & 178 & 95.2 & 771.1 & $736 \cdot 2$ & ND & ND \\
\hline & & $35-54$ years & 245 & $89 \cdot 0$ & $516 \cdot 8$ & $421 \cdot 3$ & 354 & $94 \cdot 1$ & 838.3 & 603.1 & ND & ND \\
\hline & & $55-79$ years & 188 & 89.9 & $508 \cdot 8$ & 380.1 & 308 & 92.6 & 703.9 & 604.0 & ND & ND \\
\hline & Women & $18-34$ years & 266 & $97 \cdot 0$ & 594.0 & $411 \cdot 1$ & 280 & 97.6 & $800 \cdot 0$ & 561.4 & ND & ND \\
\hline & & $35-54$ years & 270 & $96 \cdot 7$ & $593 \cdot 7$ & 378.9 & 477 & $96 \cdot 8$ & $855 \cdot 8$ & 539.8 & ND & ND \\
\hline & & $55-79$ years & 196 & $95 \cdot 9$ & $525 \cdot 4$ & 378.1 & 325 & 97.6 & $767 \cdot 1$ & 497.5 & ND & ND \\
\hline \multirow{6}{*}{ Non-alcoholic beverages } & Men & $18-34$ years & 180 & $80 \cdot 0$ & 195.5 & 229.7 & 178 & 92.4 & 333.2 & 370.9 & ND & ND \\
\hline & & $35-54$ years & 245 & $50 \cdot 6$ & $66 \cdot 1$ & 111.4 & 354 & $50 \cdot 6$ & 118.7 & 158.4 & ND & ND \\
\hline & & $55-79$ years & 188 & 33.5 & $43 \cdot 1$ & $109 \cdot 0$ & 308 & 68.6 & 87.0 & 159.1 & ND & ND \\
\hline & Women & $18-34$ years & 266 & $80 \cdot 5$ & $138 \cdot 4$ & 161.4 & 280 & 33.5 & $213 \cdot 7$ & 276.4 & ND & ND \\
\hline & & $35-54$ years & 270 & $58 \cdot 1$ & $61 \cdot 3$ & 88.5 & 477 & 54.5 & $114 \cdot 1$ & 158.8 & ND & ND \\
\hline & & $55-79$ years & 196 & 43.9 & $51 \cdot 2$ & $127 \cdot 4$ & 325 & 80.5 & 63.9 & 79.8 & ND & ND \\
\hline \multirow[t]{6}{*}{ Alcoholic beverages } & Men & $18-34$ years & 180 & $67 \cdot 2$ & $160 \cdot 2$ & 298.4 & 178 & 64.7 & 149.5 & 261.9 & ND & ND \\
\hline & & $35-54$ years & 245 & 88.2 & $317 \cdot 1$ & 306.7 & 354 & $86 \cdot 1$ & 248.0 & 248.2 & ND & ND \\
\hline & & $55-79$ years & 188 & $90 \cdot 4$ & $312 \cdot 7$ & $285 \cdot 2$ & 308 & 91.4 & 311.0 & 319.5 & ND & ND \\
\hline & Women & $18-34$ years & 266 & $61 \cdot 3$ & $65 \cdot 0$ & 111.6 & 280 & $52 \cdot 3$ & $52 \cdot 2$ & 99.0 & ND & ND \\
\hline & & $35-54$ years & 270 & $70 \cdot 7$ & 112.5 & $151 \cdot 1$ & 477 & 64.5 & $68 \cdot 1$ & 93.2 & ND & ND \\
\hline & & $55-79$ years & 196 & 68.9 & 98.6 & $126 \cdot 2$ & 325 & 73.5 & $82 \cdot 4$ & 98.0 & ND & ND \\
\hline \multirow[t]{6}{*}{ Coffee } & Men & $18-34$ years & 180 & 78.9 & $186 \cdot 2$ & 221.3 & 178 & 61.9 & $163 \cdot 8$ & 286.4 & ND & ND \\
\hline & & $35-54$ years & 245 & 91.0 & $261 \cdot 2$ & $223 \cdot 3$ & 354 & 93.8 & 369.6 & 292.0 & ND & ND \\
\hline & & $55-79$ years & 188 & 91.5 & $254 \cdot 1$ & 218.9 & 308 & 87.8 & 264.9 & 260.5 & ND & ND \\
\hline & Women & $18-34$ years & 266 & $72 \cdot 2$ & $153 \cdot 3$ & $217 \cdot 1$ & 280 & 64.6 & 190.8 & 324.2 & ND & ND \\
\hline & & $35-54$ years & 270 & $90 \cdot 0$ & 241.8 & $212 \cdot 2$ & 477 & 81.3 & $270 \cdot 4$ & 256.6 & ND & ND \\
\hline & & $55-79$ years & 196 & $91 \cdot 3$ & $198 \cdot 2$ & 173.4 & 325 & 83.7 & $232 \cdot 0$ & 283.2 & ND & ND \\
\hline \multirow[t]{6}{*}{ Other hot beverages } & Men & $18-34$ years & 180 & $42 \cdot 8$ & 31.5 & 91.3 & 178 & 56.4 & $44 \cdot 6$ & 111.1 & ND & ND \\
\hline & & $35-54$ years & 245 & $35 \cdot 1$ & 41.3 & 112.4 & 354 & 50.2 & $76 \cdot 4$ & 170.7 & ND & ND \\
\hline & & $55-79$ years & 188 & $35 \cdot 1$ & $72 \cdot 8$ & 154.5 & 308 & $49 \cdot 6$ & $90 \cdot 8$ & 189.6 & ND & ND \\
\hline & Women & $18-34$ years & 266 & 59.4 & $76 \cdot 2$ & $166 \cdot 0$ & 280 & 66.5 & $115 \cdot 6$ & 205.9 & ND & ND \\
\hline & & $35-54$ years & 270 & 47.4 & 108.4 & $226 \cdot 2$ & 477 & $68 \cdot 3$ & 214.4 & 289.5 & ND & ND \\
\hline & & $55-79$ years & 196 & $50 \cdot 0$ & $127 \cdot 1$ & 244.5 & 325 & 68.4 & 217.0 & 295.1 & ND & ND \\
\hline
\end{tabular}

Mean values were significantly different: ${ }^{*} P<0.05$; ${ }^{* *} P<0.01 ;{ }^{* \star *} P<0.001$

$\mathrm{ND}$, not determined because of methodological differences between the two survey. 
INCA2: young adults ate more meat and less eggs and fish. But age differences in meat and meat product consumption disappeared in women.

Trends in fruit and vegetable consumption. Between the INCA1 and INCA2 studies, fresh fruit consumption remained stable in younger people but increased in middle-aged adults $(+48.4 \%$ in women and $+37.8 \%$ in men) and older women $(+23.4 \%)$. Vegetable consumption was steady in middleaged adults and older men, increased in older women $(+15.0 \%)$, but decreased in young men $(-13.8 \%)$. Consequently, the overall consumption of fresh fruit and vegetables increased since the INCA1 study, particularly in the elderly $(+6.4 \%$ in men and $+19.5 \%$ in women) who had the highest intakes in 1998-99, emphasizing the age differences in consumption levels. In addition, more middle-aged $(+47.3 \%)$ and old $(+37.5 \%)$ women consumed mashed and cooked fruit in INCA2.

Trends in savoury snack food consumption. The only significant evolution observed between the two studies concerning snacking foods was the increase in pizza and savoury pastries eaten by middle-aged men $(+33.0 \%)$. The age-related trends were maintained: pizza and sandwich consumption decreased with increasing age.

\section{Trends in nutritional intake between 1998-99 and 2006-07}

Mean energy intake remained unchanged between the two studies, but macronutrient contributions to energy changed (Table 4). The energy from proteins decreased in women $(-4.4$ to $-5.1 \%)$ and middle-aged men $(-3.4 \%)$. In the elderly, the carbohydrate contribution to energy decreased $(-3.0 \%$ in women and $-4.7 \%$ in men) and the lipid component increased $(+5.6 \%$ in women and $+6.1 \%$ in men), while the inverse was observed in young men $(+3.5$ and $-4.2 \%$, respectively). The amounts of polysaccharides fell in every sex-age subgroup, whereas the sugar amounts rose significantly in young men $(+9.8 \%)$ and middle-aged women $(+12.7 \%)$ only.

Vitamin $\mathrm{C}$ and folate intakes increased and $\mathrm{Na}$ intake was reduced in every sex-age subgroup. Some sex-age-specific trends occurred: fibre and iron intakes increased in middleaged $(+11.2$ and $+8.8 \%$, respectively) and old $(+7.7$ and $-7.1 \%$ ) women, and $\mathrm{Ca}$ intake rose in middle-aged adults $(+14.2 \%$ in women and $+12.5 \%$ in men) and young men $(+8.5 \%)$.

\section{Discussion}

In France, it is the first time that two consecutive food consumption surveys with a similar methodology enabled to monitor eating habits and nutrient intake trends at a national scale. The two samples were representative of people living in France, recorded diet with the same tools (7-d food dairy) and had annual coverage to allow comparison of food group consumption and nutritional intake ${ }^{(9,10)}$. Moreover, many constraints have been followed to limit methodological bias for estimating trends. First, under-reporters were identified and excluded according to the same conditions in both surveys. Then, to ensure comparison on the same food groups, the foods recorded in the INCA1 survey were recoded according to the INCA2 food group nomenclature.
Nevertheless, there are some limitations in the present study. Concerning the identification of under-reporters, height and weight were self-reported in the INCA1 survey and directly measured in INCA2. Height and weight misreporting has often been described ${ }^{(28-30)}$. But further calculations to estimate the error in $\mathrm{BMR}^{(20)}$ resulting from weight underestimation values (as described in a recent review $^{(31)}$ ) led us to consider that weight misreporting in the INCA1 study should not have a significant impact on how under-reporters are identified. Concerning changes in food consumption, some improvements in the food records were introduced in the INCA2 survey: special emphasis was made on beverage, sauce and condiment intakes recording during survey-taker training, and questions on the addition of sugar and fat were included in the self-administered questionnaire and used to correct the records. Because of these improvements, comparisons of beverage and fat consumption or fatty acid intake were not possible and therefore not presented. As for nutritional intake, the food composition data came from the same source (CIQUAL database), dating back to the period of each survey (1998 and 2006). Additional nutritional analyses and data from the scientific literature have improved the accuracy of the food composition dataset between the two surveys, and this methodological issue might explain part of the nutritional trends observed. Nevertheless, these trends were consistent with those described in food intake; thus, improvement of the food composition data might have played only a slight role on nutritional intake trends. Moreover, industrial food product composition and the food supply have also evolved to meet the nutritional recommendations ( salt $^{(32)}$, trans fatty acids $^{(33)}$ and sugars ${ }^{(34)}$ ) based on the national nutritional policy $^{(2)}$. Therefore, keeping the food composition tables related to each survey period appeared to be the best compromise to take into account changes in the food composition. Finally, the threshold value of $P<0.05$ was maintained despite the number of tests performed. For a given food group, the trends shown were consistent within the sex-age subgroups (either increase or decrease). In addition, they were also observed in the children's sample ${ }^{(35)}$, which supported the present findings concerning French dietary intake changes.

The main findings of the present study were a decrease in the consumption of dairy products (mainly milk and cheese), meat, bread, potatoes, pastries/croissant-like pastries/cakes/ biscuits and sugar/confectionery. Conversely, the consumption of fruit and vegetables, rice, ice cream and chocolate increased. Other food groups, like fish and snacking foods, remained stable. Some 'consumer rate' and 'mean amounts eaten' trends were consistent with each other (e.g. rice, ice cream, chocolate, milk and eggs), whereas other trends involved only amounts consumed (e.g. bread, cheese and sugar). These changes took place during the establishment of the French nutritional policy. Indeed, INCA1 was completed before the First National Nutrition and Health Program $^{(2)}$ was launched, whereas INCA2 was started at the end of this first program. Although some dietary changes were consistent with the food-based dietary guidelines (increased consumption of fruit and vegetables and decreased intake of sweetened foods), many were not (stability of fish consumption and decrease in dairy product intake). 
Table 4. Trends in nutritional intakes (g/d), by sex and age, between Individual and National Food Consumption Surveys (INCA1, 1998-99 and INCA2, 2006-07) surveys (under-reporters excluded) (Mean values and standard deviations)

\begin{tabular}{|c|c|c|c|c|c|c|c|c|c|c|c|c|c|c|c|}
\hline \multirow[b]{3}{*}{ Food group } & \multirow[b]{3}{*}{ Age } & \multicolumn{6}{|c|}{ Men } & \multirow[b]{3}{*}{ Trends (\%) } & \multicolumn{7}{|c|}{ Women } \\
\hline & & \multicolumn{3}{|c|}{ INCA1 (1998-99) } & \multicolumn{3}{|c|}{ INCA2 (2006-07) } & & \multicolumn{3}{|c|}{ INCA1 (1998-99) } & \multicolumn{3}{|c|}{ INCA2 (2006-07) } & \multirow[b]{2}{*}{ Trends (\%) } \\
\hline & & $n$ & Mean & SD & $n$ & Mean & SD & & $n$ & Mean & SD & $n$ & Mean & SD & \\
\hline \multirow[t]{3}{*}{ Energy (MJ/d) } & $18-34$ years & 180 & $10 \cdot 58$ & $2 \cdot 31$ & 178 & $10 \cdot 61$ & $2 \cdot 82$ & +0.3 & 266 & $8 \cdot 20$ & 1.65 & 280 & 8.08 & 1.59 & -1.5 \\
\hline & $35-54$ years & 245 & 10.53 & $2 \cdot 36$ & 354 & 10.50 & $2 \cdot 15$ & -0.2 & 270 & 8.03 & 1.65 & 477 & $8 \cdot 13$ & 1.47 & $+1 \cdot 2$ \\
\hline & $55-79$ years & 188 & 10.02 & 2.48 & 308 & $10 \cdot 03$ & 2.46 & +0.1 & 196 & $7 \cdot 72$ & 1.71 & 325 & $7 \cdot 86$ & 1.51 & +1.8 \\
\hline \multirow[t]{3}{*}{ Carbohydrates (\% energy) } & $18-34$ years & 180 & 44.0 & 6.8 & 178 & $45 \cdot 5$ & $6 \cdot 8$ & $+3 \cdot 5^{\star}$ & 266 & $44 \cdot 3$ & 6.4 & 280 & 44.6 & $6 \cdot 2$ & +0.7 \\
\hline & $35-54$ years & 245 & 43.4 & $6 \cdot 8$ & 354 & $44 \cdot 1$ & $6 \cdot 2$ & +1.5 & 270 & $43 \cdot 1$ & $6 \cdot 8$ & 477 & $44 \cdot 1$ & $5 \cdot 3$ & $+2 \cdot 3^{\star}$ \\
\hline & $55-79$ years & 188 & $45 \cdot 7$ & $7 \cdot 4$ & 308 & 43.5 & $7 \cdot 8$ & $-4 \cdot 7^{\star \star}$ & 196 & $44 \cdot 8$ & $7 \cdot 3$ & 325 & 43.5 & $6 \cdot 0$ & $-3 \cdot 0^{*}$ \\
\hline \multirow[t]{3}{*}{ Proteins (\% energy) } & $18-34$ years & 180 & $16 \cdot 8$ & $2 \cdot 8$ & 178 & $16 \cdot 8$ & $3 \cdot 1$ & +0.5 & 266 & $16 \cdot 5$ & $2 \cdot 7$ & 280 & $15 \cdot 7$ & $2 \cdot 4$ & $-4 \cdot 9^{\star \star \star}$ \\
\hline & $35-54$ years & 245 & $17 \cdot 9$ & $2 \cdot 8$ & 354 & $17 \cdot 2$ & $2 \cdot 7$ & $-3 \cdot 4^{\star *}$ & 270 & 17.5 & 3.0 & 477 & $16 \cdot 6$ & 2.5 & $-5 \cdot 1^{* \star *}$ \\
\hline & $55-79$ years & 188 & $17 \cdot 6$ & $2 \cdot 7$ & 308 & $17 \cdot 5$ & $3 \cdot 2$ & -0.5 & 196 & $17 \cdot 5$ & $2 \cdot 7$ & 325 & $16 \cdot 7$ & $2 \cdot 4$ & $-4 \cdot 4^{\star \star \star}$ \\
\hline \multirow{3}{*}{ Lipids (\% energy) } & $18-34$ years & 180 & $39 \cdot 3$ & 5.5 & 178 & $37 \cdot 6$ & 5.4 & $-4 \cdot 2^{\star \star}$ & 266 & 39.2 & $5 \cdot 2$ & 280 & 39.7 & $5 \cdot 8$ & $+1 \cdot 3$ \\
\hline & $35-54$ years & 245 & $38 \cdot 7$ & $5 \cdot 7$ & 354 & 38.6 & $5 \cdot 3$ & -0.2 & 270 & 39.4 & 5.9 & 477 & $39 \cdot 3$ & 4.8 & -0.2 \\
\hline & $55-79$ years & 188 & $36 \cdot 7$ & $6 \cdot 6$ & 308 & 38.9 & 6.9 & $+6 \cdot 1^{\star \star \star}$ & 196 & $37 \cdot 7$ & $6 \cdot 6$ & 325 & 39.8 & 5.5 & $+5 \cdot 6^{\star \star \star}$ \\
\hline \multirow[t]{3}{*}{ Proteins (g/d) } & $18-34$ years & 180 & $101 \cdot 7$ & 24.2 & 178 & $102 \cdot 4$ & 29.8 & +0.7 & 266 & $78 \cdot 7$ & $16 \cdot 9$ & 280 & 74.0 & $16 \cdot 2$ & $-6 \cdot 0^{* \star *}$ \\
\hline & $35-54$ years & 245 & $103 \cdot 6$ & $25 \cdot 2$ & 354 & $101 \cdot 0$ & $22 \cdot 9$ & $-2 \cdot 5$ & 270 & $80 \cdot 4$ & $19 \cdot 3$ & 477 & $78 \cdot 2$ & $16 \cdot 3$ & $-2 \cdot 8$ \\
\hline & $55-79$ years & 188 & $96 \cdot 3$ & $24 \cdot 6$ & 308 & $96 \cdot 0$ & 24.9 & -0.3 & 196 & $77 \cdot 3$ & $18 \cdot 6$ & 325 & $75 \cdot 3$ & $15 \cdot 0$ & $-2 \cdot 6$ \\
\hline \multirow{3}{*}{ Carbohydrates (g/d) } & $18-34$ years & 180 & 270.9 & $76 \cdot 6$ & 178 & $279 \cdot 7$ & 91.9 & $+3 \cdot 2$ & 266 & 214.9 & 58.9 & 280 & $212 \cdot 1$ & $53 \cdot 7$ & -1.3 \\
\hline & $35-54$ years & 245 & $257 \cdot 1$ & $84 \cdot 3$ & 354 & $262 \cdot 3$ & 71.3 & +2.0 & 270 & $200 \cdot 7$ & $56 \cdot 1$ & 477 & $209 \cdot 3$ & $47 \cdot 1$ & $+4 \cdot 3^{*}$ \\
\hline & $55-79$ years & 188 & $253 \cdot 8$ & $83 \cdot 0$ & 308 & $243 \cdot 2$ & $85 \cdot 8$ & $-4 \cdot 2$ & 196 & $201 \cdot 2$ & $63 \cdot 0$ & 325 & $199 \cdot 5$ & $52 \cdot 3$ & -0.9 \\
\hline \multirow[t]{3}{*}{ Sugars (g/d) } & $18-34$ years & 180 & $108 \cdot 2$ & $46 \cdot 1$ & 178 & $118 \cdot 8$ & $55 \cdot 8$ & $+9 \cdot 8^{*}$ & 266 & $93 \cdot 6$ & $37 \cdot 4$ & 280 & 94.4 & 34.6 & +0.9 \\
\hline & $35-54$ years & 245 & $94 . \overline{7}$ & $40 \cdot 4$ & 354 & $99 \cdot 3$ & 41.8 & $+4 \cdot 8$ & 270 & 84.5 & $32 \cdot 3$ & 477 & $95 \cdot 2$ & $29 \cdot 1$ & $+12 \cdot 7^{\star \star \star}$ \\
\hline & $55-79$ years & 188 & 91.9 & $38 \cdot 0$ & 308 & $90 \cdot 1$ & $43 \cdot 3$ & $-2 \cdot 0$ & 196 & $86 \cdot 2$ & $29 \cdot 8$ & 325 & 89.7 & $29 \cdot 2$ & +4.0 \\
\hline Polysaccharides (g/d) & $18-34$ years & 180 & $162 \cdot 7$ & 53.8 & 178 & 151.5 & $57 \cdot 8$ & $-6 \cdot 9^{\star}$ & 266 & $121 \cdot 4$ & 38.9 & 280 & $111 \cdot 8$ & 34.8 & $-7 \cdot 8^{\star \star}$ \\
\hline & $35-54$ years & 245 & $162 \cdot 3$ & $66 \cdot 8$ & 354 & $155 \cdot 8$ & 48.9 & $-4 \cdot 0$ & 270 & $116 \cdot 2$ & $40 \cdot 2$ & 477 & $109 \cdot 6$ & 31.4 & $-5 \cdot 7^{\star}$ \\
\hline & $55-79$ years & 188 & 161.9 & $63 \cdot 8$ & 308 & $147 \cdot 1$ & $65 \cdot 0$ & $-9 \cdot 2^{\star *}$ & 196 & $115 \cdot 0$ & $48 \cdot 7$ & 325 & $105 \cdot 6$ & 34.9 & $-8 \cdot 2^{*}$ \\
\hline Lipids (g/d) & $18-34$ years & 180 & $106 \cdot 8$ & $27 \cdot 2$ & 178 & 101.9 & $28 \cdot 0$ & $-4 \cdot 6$ & 266 & 83.5 & $19 \cdot 0$ & 280 & 83.6 & $20 \cdot 7$ & +0.2 \\
\hline & $35-54$ years & 245 & 99.7 & 23.5 & 354 & $101 \cdot 3$ & $25 \cdot 2$ & +1.5 & 270 & $81 \cdot 0$ & $20 \cdot 8$ & 477 & $82 \cdot 9$ & $18 \cdot 6$ & $+2 \cdot 3$ \\
\hline & $55-79$ years & 188 & $89 \cdot 7$ & $27 \cdot 0$ & 308 & 95.5 & 28.5 & $+6 \cdot 5^{\star}$ & 196 & $74 \cdot 7$ & $20 \cdot 9$ & 325 & $80 \cdot 4$ & $17 \cdot 9$ & $+7 \cdot 6^{\star * *}$ \\
\hline SFA $(g / d)$ & $18-34$ years & 180 & $46 \cdot 4$ & 13.5 & 178 & 43.5 & $14 \cdot 0$ & ND & 266 & $36 \cdot 3$ & 8.8 & 280 & 34.4 & 9.9 & ND \\
\hline & $35-54$ years & 245 & 43.4 & 11.3 & 354 & $41 \cdot 8$ & $13 \cdot 7$ & ND & 270 & $35 \cdot 7$ & $10 \cdot 6$ & 477 & 33.7 & 8.8 & ND \\
\hline & $55-79$ years & 188 & $39 \cdot 7$ & $12 \cdot 9$ & 308 & 38.6 & $14 \cdot 1$ & ND & 196 & 32.9 & $10 \cdot 2$ & 325 & $31 \cdot 6$ & 8.8 & ND \\
\hline MUFA (g/d) & $18-34$ years & 180 & $37 \cdot 8$ & $10 \cdot 0$ & 178 & $36 \cdot 1$ & 11.4 & ND & 266 & $29 \cdot 7$ & $7 \cdot 1$ & 280 & $30 \cdot 0$ & $8 \cdot 2$ & ND \\
\hline & $35-54$ years & 245 & 35.5 & 8.4 & 354 & $36 \cdot 1$ & 9.5 & ND & 270 & $28 \cdot 7$ & $7 \cdot 3$ & 477 & 29.5 & 7.5 & ND \\
\hline & $55-79$ years & 188 & 31.4 & 9.9 & 308 & $34 \cdot 0$ & 11.5 & ND & 196 & $26 \cdot 2$ & $7 \cdot 9$ & 325 & $29 \cdot 3$ & 8.0 & ND \\
\hline PUFA (g/d) & $18-34$ years & 180 & $11 \cdot 7$ & 3.9 & 178 & $13 \cdot 2$ & 5.5 & ND & 266 & $9 \cdot 1$ & $3 \cdot 6$ & 280 & $12 \cdot 3$ & $5 \cdot 0$ & ND \\
\hline & $35-54$ years & 245 & $10 \cdot 8$ & $4 \cdot 1$ & 354 & 14.5 & $5 \cdot 2$ & ND & 270 & $8 \cdot 7$ & 3.0 & 477 & $12 \cdot 9$ & 4.9 & ND \\
\hline & $55-79$ years & 188 & $9 \cdot 7$ & 3.9 & 308 & 14.9 & $7 \cdot 4$ & ND & 196 & 8.3 & $4 \cdot 1$ & 325 & 13.0 & 4.8 & ND \\
\hline Fibre (g/d) & $18-34$ years & 180 & $17 \cdot 8$ & $5 \cdot 6$ & 178 & $16 \cdot 9$ & 5.9 & $-5 \cdot 4$ & 266 & 14.5 & $4 \cdot 3$ & 280 & $14 \cdot 1$ & $4 \cdot 1$ & $-2 \cdot 8$ \\
\hline & $35-54$ years & 245 & $19 \cdot 1$ & $6 \cdot 1$ & 354 & $19 \cdot 0$ & $7 \cdot 1$ & -0.3 & 270 & $15 \cdot 2$ & 4.6 & 477 & $17 \cdot 0$ & $5 \cdot 0$ & $+11 \cdot 2^{\star \star \star}$ \\
\hline & $55-79$ years & 188 & $20 \cdot 8$ & $7 \cdot 1$ & 308 & $19 \cdot 9$ & $7 \cdot 4$ & $-4 \cdot 2$ & 196 & $16 \cdot 9$ & 5.5 & 325 & $18 \cdot 2$ & 4.9 & $+7 \cdot 7^{\star \star}$ \\
\hline Alcohol (g/d) & $18-34$ years & 180 & 11.5 & $19 \cdot 4$ & 178 & $12 \cdot 7$ & $21 \cdot 0$ & +10.4 & 266 & $5 \cdot 3$ & 8.6 & 280 & 4.4 & 8.0 & $-15 \cdot 5$ \\
\hline & $35-54$ years & 245 & $25 \cdot 2$ & 24.4 & 354 & $20 \cdot 4$ & $20 \cdot 3$ & $-18 \cdot 9^{\star \star}$ & 270 & $9 \cdot 6$ & $13 \cdot 0$ & 477 & $6 \cdot 1$ & $8 \cdot 2$ & $-36 \cdot 2^{\star \star \star}$ \\
\hline & $55-79$ years & 188 & $26 \cdot 9$ & 24.4 & 308 & 25.4 & $23 \cdot 2$ & $-5 \cdot 8 \%$ & 196 & 8.6 & 11.0 & 325 & $7 \cdot 3$ & 8.6 & $-16 \cdot 0$ \\
\hline $\mathrm{Na}(\mathrm{mg} / \mathrm{d})$ & $18-34$ years & 180 & $3527 \cdot 1$ & $1131 \cdot 8$ & 178 & $3292 \cdot 3$ & 1194.0 & $-6 \cdot 7^{*}$ & 266 & $2672 \cdot 6$ & 751.4 & 280 & $2637 \cdot 8$ & 853.3 & -1.3 \\
\hline & $35-54$ years & 245 & $3684 \cdot 8$ & 1175.9 & 354 & 3473.5 & 958.2 & $-5 \cdot 7^{\star}$ & 270 & $2705 \cdot 7$ & 848.3 & 477 & 2591.0 & $652 \cdot 3$ & $-4 \cdot 2^{*}$ \\
\hline & $55-79$ years & 188 & $3704 \cdot 1$ & $1245 \cdot 5$ & 308 & 3428.7 & $1228 \cdot 8$ & $-7 \cdot 4^{\star}$ & 196 & 2834.2 & $967 \cdot 1$ & 325 & $2632 \cdot 7$ & 754.4 & $-7 \cdot 1^{\star \star}$ \\
\hline $\mathrm{Ca}(\mathrm{mg} / \mathrm{d})$ & $18-34$ years & 180 & 933.8 & 331.5 & 178 & $1013 \cdot 6$ & $410 \cdot 6$ & $+8 \cdot 5^{\star}$ & 266 & $827 \cdot 1$ & 267.9 & 280 & 833.6 & $262 \cdot 8$ & +0.8 \\
\hline
\end{tabular}


Food choices are mostly age specific, and these age differences remain consistent over the years. In both studies, the elderly presented a more traditional diet composed of bread, fish, vegetables, fruit, sugar/confectionery, soups, cheese (men only) and mashed/cooked fruit (women only). Young adults preferred pasta, rice, croissant-like pastries/other sweetened pastries/cakes/biscuits, milk, chocolate, pizzas, sandwiches, mixed dishes, meat (men only), cream desserts (men only) and ice cream (women only), resulting in a diet that could be labelled 'snacking and convenient'. This distribution by age was concordant with previous results ${ }^{(8)}$. Other agespecific food habits changed during the 8 years interval (e.g. eggs, meat products, dairy products other than milk and cheese in both sexes, as well as cheese, meat, potatoes and cream desserts in women). The limitation of two time points makes it difficult to state whether these food habits were generational or simply a factor of age. The next INCA study will enable further analysis of age-specific food habits to determine those that are generational and will disappear within the French diet. The Research Centre for the Study and the Observation of Living Conditions already published a study on generational, age and period effects on French food trends ${ }^{(36)}$. Based on INSEE data, the age-specific trends in food consumption observed in that study were in accordance with the present findings. Many of them showed a generational effect (fruit, vegetables, potatoes, bread, fish and beef), meaning that their consumption decreases in later generations.

No data from national dietary surveys are available for comparison, but the present results are consistent with the French food balance sheet data published by the INSEE ${ }^{(37)}$. Indeed, INSEE data showed that between 1998 and 2005, the availability of bread $(-7 \%)$, meat $(-7 \%)$, milk $(-20 \%)$ and sugar/confectionery $(-12 \%)$ decreased, while that of fruit $(+10 \%)$ and rice $(+53 \%)$ increased, and that of vegetables $(+2 \%)$ and cheese $(-2 \%)$ remained stable. The global coherence in trends observed from the two datasets indicates accurate prediction of changes in French dietary intake. Moreover, a study in middle-aged people ${ }^{(38)}$ in Eastern France monitoring trends in food consumption between 1987 and 1997 already showed a similar decline in the consumption of meat, eggs and high-fat dairy products (cheese). However, other trends were different: increased consumption of fish, poultry and low-fat dairy products, and stability of fruit and vegetable consumption. The different periods of study (1987-97 v. 1998-2007) or regional trends, which are very strong and persistent within France ${ }^{(8)}$, could explain these differences.

Trends in other European countries require careful comparison as food survey methodologies and dietary habits diverge. Southern countries have been traditionally described as consuming mostly vegetal foods (pulses, fruit and vegetables and cereals) and fish, and Northern countries as consuming animal products (butter, milk and dairy products, eggs and meats) and soft drinks ${ }^{(39,40)}$. Nevertheless, the traditional diets of Mediterranean and Northern countries are converging towards a more Western diet ${ }^{(6,7,40)}$. For example, Mediterranean countries have increased their consumption of animal products (meats, milk and dairy products) and decreased wine and cereal intakes, with some components of the traditional diet still remaining (olive oil, fruit and vegetables) $)^{(7,12,41)}$. Northern countries show increased intakes of 
fruit, vegetables and cereal-based products, and decreases in meat, meat products, eggs, potatoes and sometimes sweetened foods and soft drink ${ }^{(3,15)}$. These trends toward healthier dietary habits in Northern countries and especially Scandinavian countries occurred because of strong national nutrition policies $^{(3,5)}$. The intermediate geographical position of France within Europe is reflected in its foods habits, also intermediate between Northern and Mediterranean diets: in the INCA2 survey $^{(35)}$, Northern French inhabitants ate more butter, margarine, potatoes and pastries than their Southern counterparts, who preferred oils and vegetables. Consequently, the average French diet is characterised by a relatively high consumption of fruit and vegetables, wine, fish, but also of animal products (dairy products, meat and butter) ${ }^{(6,40,41)}$. The trends in food consumption underlined in the present study were quite similar to those in Northern countries: stability of fish and cerealbased foods; increase in fruit and vegetables; decrease in meat, dairy product and sweetened food consumption. But differences in the initial amounts eaten put French dietary behaviour in an intermediate position in Europe.

Changes in cost constraints could also influence food choices $^{(42,43)}$. INSEE data show that overall foodstuff costs increased by $17.3 \%$ between 1998 and 2006, with important disparities between food categories. Thus, bread $(+20.6 \%)$ and red meat $(+26.4$ to $+33.4 \%)$ showed the highest increases, whereas the cost of cereal-based products $(+6.8 \%)$, dairy products $(+6.7 \%)$, eggs $(+5.6 \%)$ and ice cream $(+6.8 \%)$ remained relatively stable over the 8 -year period $^{(44)}$. Price trends might explain some of the dietary changes observed, with decreased consumption when prices rose (e.g. bread and meats) and vice versa (e.g. cereals, dairy products and ice cream).

Differences in the distribution of occupational status could influence food consumption ${ }^{(45-47)}$. Since the samples were nationally representative, they partly reflected the socioeconomic evolution of the French population, as confirmed by the INSEE data. Nevertheless, adjusting consumption by individual occupational status did not change the overall trends (results not shown).

Changing trends in nutritional intake between the two surveys could partly be explained by several facts: (1) changes in food consumption, (2) methodological improvements in the recording of fat and sugar intakes in INCA2 and (3) improvement in composition tables between the two surveys, as well as true changes in food composition since $1999^{(48)}$. The decrease in meat, dairy product and bread consumption in women could explain the decline in their protein intake and energy contribution. Likewise, the rise in fruit and vegetable consumption probably accounted for the increased intakes in fibre, vitamin $\mathrm{C}$ and folate. The overall decrease in polysaccharide intake could be related to the decrease in bread and potato intake, which was not balanced by increased consumption of rice and pasta. Decreased $\mathrm{Na}$ intake could be caused by decreased bread consumption, as it is the primary $\mathrm{Na}$ vector in the French $\operatorname{diet}^{(32)}$, as well as efforts by food manufacturers to reduce the salt content of their products (soups, cheese, meat products and ready-to-eat meals) to comply with the AFSSA's recommendations ${ }^{(32)}$. The increase in sugar intake was observed along with fruit and soft drink consumption increases, but methodological changes in INCA2 make a direct comparison impossible. Finally, Ca intake increased despite the drop in dairy product intake, which could be partly attributed to improvements of the nutritional composition of mineral water.

\section{Conclusion}

Several changes in the French diet have occurred since 1998-99. These changes are similar to those in Northern European countries and have tended towards an average European diet at the crossroads of Mediterranean and Northern diets. This global uniformisation towards a typical Western-type diet was more pronounced in young adults than in the elderly, who retained a more traditional French diet. Moreover, trends in food habits changed nutritional intake only slightly.

\section{Acknowledgements}

The INCA1 survey was supported by a grant from the Ministries for Health, Agriculture and Consumer Affairs and INCA2 by a grant from the French Food Safety Agency (AFSSA). The authors wish to thank the CIQUAL group for providing the national food composition tables, the Institut de Sondage Lavialle for the collection of data and all the families for their cooperation. C. D. analysed the data and drafted the paper; A. D., G. C-.T. and J-.L. V. contributed to the design and data collection of both INCA surveys; and C. D., S. L., M. T. and L. L. contributed to the design and data collection of the INCA2 survey. A. D., J-.L. V., S. L., M. T. and L. L. assisted in the drafting of the paper. All the authors reviewed the manuscript. There are no conflicts of interest in the preparation of the paper.

\section{References}

1. WHO \& FAO (2003) Diet, Nutrition and the Prevention of Chronic Diseases. Report of a Joint WHO/FAO Expert Consultation. Report no. 916. Geneva: WHO.

2. Hercberg S, Chat-Yung S \& Chauliac M (2008) The French National Nutrition and Health Program: 2001-2006-2010. Int J Public Health 53, 68-77.

3. Prattala R (2003) Dietary changes in Finland - success stories and future challenges. Appetite 41, 245-249.

4. Kennedy E (2008) Nutrition policy in the US: 50 years in review. Asia Pac J Clin Nutr 17, Suppl. 1, 340-342.

5. Kaernes U (2003) Experiences with the Norwegian nutrition policy. Appetite 41, 251-257.

6. Schmidhuber J \& Traill WB (2006) The changing structure of diets in the European Union in relation to healthy eating guidelines. Public Health Nutr 9, 584-595.

7. Balanza R, Garcia-Lorda P, Perez-Rodrigo C, et al. (2007) Trends in food availability determined by the Food and Agriculture Organization's food balance sheets in Mediterranean Europe in comparison with other European areas. Public Health Nutr 10, 168-176.

8. Kesse E, Boutron-Ruault MC \& Clavel-Chapelon F (2005) Regional dietary habits of French women born between 1925 and 1950. Eur J Nutr 44, 285-292.

9. Champagne CM, Bogle ML \& Karge WH (2002) Using national dietary data to measure dietary changes. Public Health Nutr $\mathbf{5}$, 985-989.

10. Dubuisson C, Lioret S, Gautier A, et al. (2006) Comparison of two national food surveys (INCA 1 1998-99 and Health Nutrition Barometer 2002) with regard to five food recommendations 
of the National Nutrition and Health Program. Rev Epidemiol Sante Publique 54, 5-14.

11. Ribas-Barba L, Serra-Majem L, Salvador G, et al. (2007) Trends in dietary habits and food consumption in Catalonia, Spain (1992-2003). Public Health Nutr 10, 1340-1353.

12. Marques-Vidal P, Ravasco P, Dias CM, et al. (2006) Trends of food intake in Portugal, 1987-1999: results from the National Health Surveys. Eur J Clin Nutr 60, 1414-1422.

13. Krachler B, Eliasson MC, Johansson I, et al. (2005) Trends in food intakes in Swedish adults 1986-1999: findings from the Northern Sweden MONICA (Monitoring of Trends and Determinants in Cardiovascular Disease) Study. Public Health Nutr 8 , 628-635.

14. Hulshof KF, Brussaard JH, Kruizinga AG, et al. (2003) Socioeconomic status, dietary intake and $10 \mathrm{y}$ trends: The Dutch National Food Consumption Survey. Eur J Clin Nutr 57, 128-137.

15. Osler M, Heitmann BL \& Schroll M (1997) Ten year trends in the dietary habits of Danish men and women. Cohort and crosssectional data. Eur J Clin Nutr 51, 535-541.

16. Kant AK \& Graubard BI (2006) Secular trends in patterns of self-reported food consumption of adult Americans: NHANES 1971-1975 to NHANES 1999-2002. Am J Clin Nutr 84, $1215-1223$.

17. Lioret S, Touvier M, Lafay L, et al. (2008) Dietary and physical activity patterns in French children are related to overweight and socioeconomic status. $J$ Nutr 138, 101-107.

18. Volatier J-L (2000) Enquête INCA individuelle et nationale sur les consommations alimentaires. (Individual and National Food Consumption INCA Survey). Paris: Tec\&Doc.

19. Deville J-C (1991) A theory of quota surveys. Survey Methodol 17, 163-181.

20. Schofield WN (1985) Predicting basal metabolic rate, new standards and review of previous work. Hum Nutr Clin Nutr 39, 5-41.

21. Black AE (2000) Critical evaluation of energy intake using the Goldberg cut-off for energy intake:basal metabolic rate. A practical guide to its calculation, use and limitations. Int $J$ Obes Relat Metab Disord 24, 1119-1130.

22. Swan G (2004) Findings from the latest National Diet and Nutrition Survey. Proc Nutr Soc 63, 505-512.

23. Mirmiran P, Esmaillzadeh A \& Azizi F (2006) Under-reporting of energy intake affects estimates of nutrient intakes. Asia Pac $J$ Clin Nutr 15, 459-464.

24. Johansson L, Solvoll K, Bjorneboe GE, et al. (1998) Under- and overreporting of energy intake related to weight status and lifestyle in a nationwide sample. Am J Clin Nutr 68, 266-274.

25. Hercberg S, Deheeger M \& Preziosi P (editors) (1994) SU-VI-MAX. Portions alimentaires. Manuel photos pour l'estimation des quantités. (Portion Sizes: Picture Booklet for the Estimation of Quantities). Paris: Polytechnica.

26. Favier J-C, Ireland-Ripert J, Toque C, et al. (1995) Répertoire général des aliments - Table de composition. (Régal French Food Composition Table), 2nd ed. Paris: Lavoisier.

27. Table de composition nutritionnelle des aliments Ciqual. (French Food Composition Table - CIQUAL). http://www. afssa.fr/TableCIQUAL/ (accessed January 2009).

28. Engstrom JL, Paterson SA, Doherty A, et al. (2003) Accuracy of self-reported height and weight in women: an integrative review of the literature. J Midwifery Women Health 48, 338-345.

29. Niedhammer I, Bugel I, Bonenfant S, et al. (2000) Validity of self-reported weight and height in the French GAZEL cohort. Int $\mathrm{J}$ Obes Relat Metab Disord 24, 1111-1118.

30. Sahyoun NR, Maynard LM, Zhang XL, et al. (2008) Factors associated with errors in self-reported height and weight in older adults. J Nutr Health Aging 12, 108-115.
31. Gorber SC, Tremblay M, Moher D, et al. (2007) A comparison of direct vs. self-report measures for assessing height, weight and body mass index: a systematic review. Obes Rev 8, 307-326.

32. AFSSA (2002) Rapport Sel: Evaluation et recommendations. (Report on Salt: Evaluation and Recommendations). Paris: Agence française de sécurité sanitaire des aliments. http://www. afssa.fr/Documents/NUT-Ra-Sel-.pdf (accessed January 2009).

33. AFSSA (2005) Risques et bénéfices, pour la santé, des acides gras trans apportés par les aliments: recommandations. (Risks and Health Benefits of trans Fatty Acids Obtained from Foods: Recommendations). Paris: Agence française de sécurité sanitaire des aliments. http://www.afssa.fr/Documents/NUT-Ra-AGtrans. pdf (accessed June 2009).

34. AFSSA (2004) Glucides et santé: Etat des lieux, évaluation et recommandations. (Carbohydrates and Health: Current Status, Evaluations and Recommendations). Paris: Agence française de sécurité sanitaire des aliments. http://www.afssa. fr/Documents/NUT-Ra-Glucides.pdf (accessed June 2009).

35. AFSSA (2009) Summary of the report of the 2006/2007 Individual and National Survey on Food Consumption 2 (INCA 2). http:// www.afssa.fr/Documents/PASER-Sy-INCA2EN.pdf.

36. Recours F, Hebel P \& Gaignier C (2005) Exercice d'anticipation des comportements alimentaires des Français - Modèle Age Période - Cohorte. (Exercise of Anticipation of French Eating Behaviours - Age-Period-Cohort Model). Report no. 222. Paris: CREDOC.

37. INSEE (2007) National accounts, base 2000 (Comptabilité nationale, base 2000). In: Statistical Yearbook of France (Annuaire statistique de la France). http://www.insee. $\mathrm{fr} / \mathrm{fr} /$ publications-et-services/sommaire.asp?id $=178 \&$ nivgeo $=0$ (accessed January 2009). Paris: INSEE.

38. Perrin AE, Simon C, Hedelin G, et al. (2002) Ten-year trends of dietary intake in a middle-aged French population: relationship with educational level. Eur J Clin Nutr 56, 393-401.

39. Trichopoulou A, Naska A \& Costacou T (2002) Disparities in food habits across Europe. Proc Nutr Soc 61, 553-558.

40. Slimani N, Fahey M, Welch AA, et al. (2002) Diversity of dietary patterns observed in the European Prospective Investigation into Cancer and Nutrition (EPIC) project. Public Health Nutr $\mathbf{5}$, $1311-1328$.

41. Garcia-Closas R, Berenguer A \& Gonzalez CA (2006) Changes in food supply in Mediterranean countries from 1961 to 2001. Public Health Nutr 9, 53-60.

42. Drewnowski A \& Darmon N (2005) Food choices and diet costs: an economic analysis. J Nutr 135, 900-904.

43. Darmon N, Ferguson EL \& Briend A (2006) Impact of a cost constraint on nutritionally adequate food choices for French women: an analysis by linear programming. J Nutr Educ Behav 38, 82-90.

44. INSEE (2008) Statistical indices and series. http://www.indices. insee.fr/bsweb/servlet/bsweb?action=BS_RECHGUIDEE\&BS_ IDARBO $=06020000000000$ (accessed January 2009).

45. Irala-Estevez JD, Groth M, Johansson L, et al. (2000) A systematic review of socio-economic differences in food habits in Europe: consumption of fruit and vegetables. Eur J Clin Nutr 54, 706-714.

46. Lallukka T, Laaksonen M, Rahkonen O, et al. (2007) Multiple socio-economic circumstances and healthy food habits. Eur $J$ Clin Nutr 61, 701-710.

47. Turrell G \& Kavanagh AM (2006) Socio-economic pathways to diet: modelling the association between socio-economic position and food purchasing behaviour. Public Health Nutr 9, 375-383.

48. Anderson E, Perloff B, Ahuja J, et al. (2001) Tracking nutrient changes for trends analysis in the United States. Journal Food Compos Anal 14, 287-294. 